Collection SFN 11 (2010) 17-42

(C) Owned by the authors, published by EDP Sciences, 2010

DOI: $10.1051 / \mathrm{sfn} / 201011003$

\title{
Introduction à la diffusion aux petits angles
}

\author{
F. Nallet \\ Université Bordeaux-1 CNRS, Centre de Recherche Paul-Pascal, 115 avenue du \\ Docteur-Schweitzer, 33600 Pessac, France
}

\begin{abstract}
Résumé. L'objectif de ce cours est de donner quelques notions de base dans l'interprétation d'une expérience de diffusion aux petits angles: principe de la diffusion (élastique et cohérente), facteur de forme et facteur de structure, régime de Guinier, régime intermédiaire, limite de Porod. La détermination du rayon de giration, du second coefficient du viriel sera abordée. Des modélisations «simples» de courbes de diffusion seront présentées avec l'introduction des facteurs de forme de sphères, de disques, de bâtonnets, de chaînes gaussiennes et d'agrégats à la géométrie fractale. Quelques applications aux cristaux-liquides permettront d'évoquer les spécificités des systèmes denses ou ordonnés. Quelques commentaires seront donnés sur les possibilités de variation de contraste offertes par la deutériation, ainsi que sur les mérites comparés de la diffusion des rayons $\mathrm{X}$ ou des neutrons aux petits angles.
\end{abstract}

\section{INTRODUCTION ET GÉNÉRALITÉS}

De quel phénomène s'agit-il lorsque l'on parle de diffusion des neutrons ou, peut-être, de rayons X, de lumière? D'un cas spécifique d'interaction rayonnement - matière, où les phénomènes d'absorption (par ex. exploités en neutrographie, en imagerie médicale aux rayons $\mathrm{X}$, en spectrophotométrie d'absorption), d'émission avec changement de fréquence (par ex. en spectroscopie de fluorescence ou en spectroscopie Raman, phénomènes également qualifiés de diffusion inélastique), ou d'un simple changement dans la direction de propagation (par ex. en imagerie optique) sont réputés négligeables. Par contraste avec les exemples précédents, il y a diffusion - dite alors «élastique» - lorsque du rayonnement est réémis par un échantillon irradié, sans dissipation globale d'énergie, sans changement notable de fréquence et $a$ priori dans toutes les directions de l'espace. Ce phénomène est aussi connu en tant que diffusion Rayleigh, dans le cas de la lumière visible, ou diffusion Thomson dans le cas des rayons X.

Alors que les phénomènes d'absorption sont souvent associés à la présence d'un élément spécifique dans l'échantillon, que la diffusion inélastique requiert la résonance d'un oscillateur atomique, moléculaire ou supramoléculaire, ce qui rend ces techniques utiles dans le domaine de l'analyse chimique, notamment, la diffusion élastique trouve son origine dans les seules hétérogénéités de propriétés physiques: son interprétation donnera donc des informations sur la structure de l'échantillon d'un point de vue, disons, géométrique. La couleur blanche du lait, ou l'éblouissement que provoque le brouillard illuminé par les feux de route d'une automobile, la nuit, proviennent d'une intense diffusion Rayleigh de la lumière par les hétérogénéités que forment les gouttelettes d'un liquide en suspension, soit dans un autre liquide (cas d'une émulsion comme le lait), soit dans un gaz (cas d'un aérosol comme le brouillard). Il est également bien connu que la luminosité diurne du ciel (et, indirectement, sa couleur bleue - vide infra Section 1.1) est la conséquence des hétérogénéités de densité de l'atmosphère, un gaz dont la densité fluctue sensiblement du seul fait qu'un tel milieu est facilement compressible.

La diffusion dont il est question dans ce chapitre est de la diffusion aux petits angles. Elle est à distinguer de la diffusion (dite aussi, pour simplifier la nomenclature, diffraction, voire réflexion ....) de

This is an Open Access article distributed under the terms of the Creative Commons Attribution-Noncommercial License 3.0, which permits unrestricted use, distribution, and reproduction in any noncommercial medium, provided the original work is properly cited. 
Bragg: cette dernière se produit lorsque les hétérogénéités en cause ont une structure périodique dans au moins une direction de l'espace, comme c'est le cas avec les matériaux ordonnés que sont les cristaux liquides smectiques ou colonnaires et les solides cristallins. La cristallographie - un vaste domaine qui ne sera pas abordé - a notamment pour objet d'interpréter, en termes essentiellement géométriques, les spectres de diffusion de Bragg. La diffusion aux petits angles est donc produite par des hétérogénéités a priori non périodiques et, qui plus est, souvent fluctuantes du fait de l'inévitable agitation thermique présente dans tout échantillon. Dernière précision de nomenclature, dans le contexte de la physicochimie des milieux colloïdaux, la diffusion aux petits angles est également connue, quand il s'agit de lumière, sous le nom de diffusion Tyndall.

\section{1 Éléments de théorie}

Pour l'étude de la matière condensée, la diffusion aux petits angles se pratique essentiellement avec le rayonnement électromagnétique (lumière visible, rayons X) et, bien sûr, les neutrons. Dans ses grandes lignes, le phénomène de diffusion est d'ailleurs largement indépendant de la nature du rayonnement employé.

Tout rayonnement est associé à un champ, une grandeur définie localement dans l'espace et le temps, dont la propagation dans le vide ou dans un milieu matériel est régie par des équations d'onde. Dans le cas de la lumière ou des rayons $X$, il s'agit du champ électrique et du champ magnétique, décrits par les équations de Maxwell. Dans le cas des neutrons, il s'agit de l'amplitude de probabilité de présence de la mécanique quantique, décrite par l'équation de Schrödinger. Il suffira ici de savoir que ces équations admettent, lorsque le milieu de propagation est strictement homogène et en l'absence de toute absorption, des solutions particulières remarquables analogues à

$$
\psi(\boldsymbol{x}, t)=\psi_{0} \exp [i(\boldsymbol{k} \cdot \boldsymbol{x}-\omega t)]
$$

où $\psi_{0}$, amplitude de l'onde, caractérise l'énergie (constante puisqu'il n'y a pas d'absorption) véhiculée par le rayonnement, $\boldsymbol{k}$, vecteur d'onde, la direction le long de laquelle le rayonnement se propage, ainsi que la fréquence de l'oscillation spatiale et enfin $\omega$, pulsation, la fréquence de l'oscillation temporelle, également parfois interprétée comme caractéristique de l'énergie d'une particule individuelle - photon ou neutron - associée au rayonnement. La longueur d'onde du rayonnement est définie comme étant $\lambda=2 \pi / k$, où $k$ note le module du vecteur d'onde. La fréquence $f$ est, quant à elle, donnée par $f=\omega /(2 \pi)$. Parce que l'onde est solution d'une équation de propagation, module du vecteur d'onde et pulsation sont de surcroît reliés par une relation dite « de dispersion », de la forme

$$
\omega=\frac{c}{n} k
$$

dans le cas du rayonnement électromagnétique et

$$
\hbar \omega=\frac{\hbar^{2} k^{2}}{2 M_{n}}+V
$$

dans le cas des neutrons.

Dans l'éq. (1.2), $c$ est la vitesse de le lumière dans le vide et $n$ l'indice de réfraction $(n=1$ pour le vide, $n$ très peu différent de 1 dans l'air) du milieu de propagation. Dans l'éq. (1.3), $\hbar$ est la constante de Planck divisée par $2 \pi, M_{n}$ la masse du neutron et $V$ l'énergie potentielle du neutron $(V=0$ dans le vide) dans le milieu de propagation. Il peut être utile d'avoir une idée des ordres de grandeur de quelques unes des grandeurs concernées, cf. tableau 1.

Lorsque le milieu de propagation est strictement homogène, à l'exception d'une singularité ponctuelle, par exemple localisée à l'origine en $\mathbf{0}$, les équations d'onde ont également des solutions particulières remarquables connues sous le nom d'ondes sphériques, dont la structure, très proche de 
Tableau 1. Ordres de grandeur typiques dans l'étude de la matière molle.

\begin{tabular}{l||c|c|c} 
& Lumière & Rayons $\mathrm{X}$ & Neutrons \\
\hline \hline$\lambda[\mathrm{m}]$ & $4-810^{-7}$ & $10^{-10}$ & $610^{-10}$ \\
\hline$f[\mathrm{~Hz}]$ & $10^{15}$ & $10^{18}$ & $10^{12}$ \\
\hline$n$ & $1,3-1,5$ & $1-\epsilon$ &
\end{tabular}

celle de l'onde plane évoquée plus haut, éq. (1.1), est la suivante

$$
\psi(\boldsymbol{x}, t)=\frac{\Psi_{0}}{R} \exp [i(k R \pm \omega t)]
$$

Dans cette expression, $R$ représente la distance entre la singularité ponctuelle et le point d'observation localisé en $\boldsymbol{x}$, c'est-à-dire $R=\|\boldsymbol{x}\|$ et $\Psi_{0}$ est une mesure de l'amplitude de l'onde sphérique. La grandeur $k$ (que l'on a coutume d'appeler «module du vecteur d'onde») et la pulsation $\omega$ restent reliées par la même relation de dispersion que dans le cas de l'onde plane, éq. (1.2) pour le rayonnement électromagnétique, éq. (1.3) pour des neutrons.

Il convient de souligner les différences - essentielles - entre l'onde sphérique et l'onde plane:

1. Alors que l'onde plane se propage dans une direction bien définie, celle du vecteur d'onde $\boldsymbol{k}$, l'onde sphérique n'a pas une unique direction de propagation. L'équation (1.4) représente un rayonnement qui est émis par une source ponctuelle, de façon isotrope dans l'espace, lorsque le terme de phase est écrit avec le signe «moins » - l'onde sphérique est alors dite « divergente »-, ou qui converge vers un point lorsque le signe «plus» est choisi - onde sphérique convergente. Une flamme de bougie ou le filament d'une lampe à incandescence, vus de suffisamment loin pour être raisonnablement assimilés à des objets ponctuels, produisent un rayonnement qui peut être décrit en termes d'ondes sphériques divergentes. Ce sont effectivement des sources de lumière qui éclairent dans toutes les directions. De même, le rayonnement au voisinage du foyer d'une lentille convergente a une certaine similitude avec l'onde sphérique convergente;

2. Alors que l'onde plane véhicule une densité d'énergie constante, la densité d'énergie dans l'onde sphérique décroît lorsque l'on s'éloigne de la singularité (et bien sûr augmente lorsque l'on s'en rapproche). Cela étant, l'énergie reste conservée en ce sens que l'énergie totale qui traverse une coquille sphérique centrée sur la singularité est indépendante du rayon $R$ de ladite coquille. En ce sens, l'onde, qui se propage au sein d'un milieu non absorbant, n'est pas atténuée.

Une bonne façon de se représenter, semi-quantitativement, le phénomène de la diffusion élastique est la suivante : parce qu'il a été «conditionné» (par le monochromateur, des dispositifs de collimation, etc.) pour qu'il en soit raisonnablement proche, le rayonnement incident qui traverse l'échantillon peut être vu, aux hétérogénéités près de l'échantillon, comme une onde plane. Chaque point d'hétérogénéité dans l'échantillon prélève une partie de l'énergie apportée par le rayonnement incident pour restituer la totalité de ce prélèvement sous la forme d'une onde sphérique divergente, à la même fréquence que celle de l'onde incidente - la diffusion est élastique. Enfin, le rayonnement diffusé est constitué de la superposition, et donc de l'interférence en diffusion cohérente, de toutes ces ondelettes sphériques.

Du fait de la vitesse finie de propagation du rayonnement, les hétérogénéités spatialement distinctes dans l'échantillon seront irradiées par l'onde incidente avec des phases variables, et le rayonnement réémis par les hétérogénéités parviendra avec un retard variable à un point donné de l'espace. Le rayonnement diffusé contient donc en principe une certaine information sur la structure spatiale des hétérogénéités de l'échantillon. Un autre point important est que toutes les hétérogénéités ne prélèvent pas avec la même efficacité de l'énergie à l'onde incidente. Il en résulte que toutes les ondelettes qui interfèrent n'ont pas la même amplitude. Le rayonnement diffusé contient donc aussi une certaine information sur l'efficacité de la conversion onde plane $\rightarrow$ onde sphérique, en rapport avec le contraste de l'échantillon, cf. Section 1.2. 


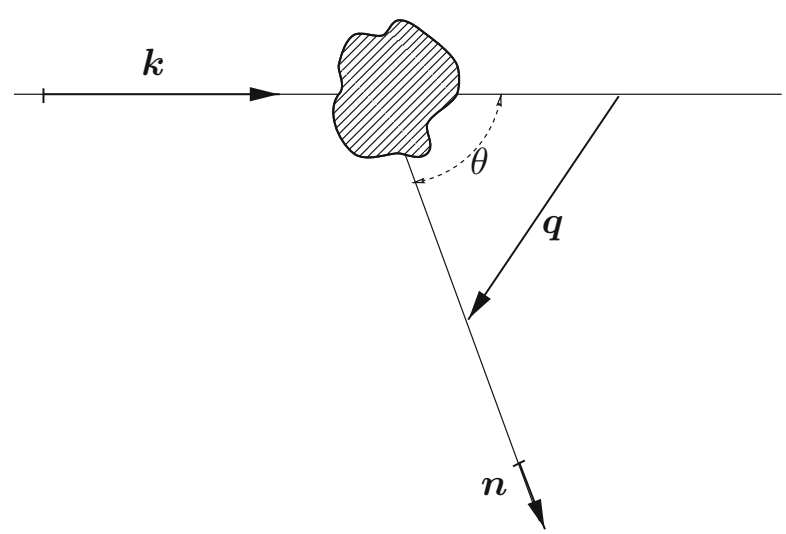

Figure 1. Schéma de principe d'une expérience de diffusion. Le rayonnement incident est une onde plane se propageant dans la direction du vecteur d'onde $\boldsymbol{k}$. L'échantillon irradié émet du rayonnement diffusé dans toutes les directions de l'espace. Une direction particulière d'observation, celle dans laquelle un détecteur est placé, par exemple, est figurée par le vecteur unitaire $\boldsymbol{n}$ qui forme un angle $\theta$ avec la direction incidente.

La traduction en termes quantitatifs des idées exposées ci-dessus repose sur une approximation, dite «de Born» ou encore de diffusion simple (par opposition à la diffusion multiple), où l'on suppose que les hétérogénéités du milieu de propagation du rayonnement restent «petites». Si le rayonnement incident est décrit par l'éq. (1.1) et que le milieu de propagation ne comporte de (petites) hétérogénéités que dans le volume fini de l'échantillon, le rayonnement diffusé à grande distance $R$ de l'échantillon, dans une direction repérée par le vecteur unitaire $\boldsymbol{n}$ (cf. Fig. 1) a pour expression

$$
\psi_{d}(R \boldsymbol{n}, t)=\psi_{0} \frac{\exp [i(k R-\omega t)]}{R} \int_{V_{\mathrm{irr}}} d^{3} x \exp [-i \boldsymbol{q} \cdot \boldsymbol{x}] h(\boldsymbol{x}, t)
$$

et s'apparente donc à l'onde sphérique divergente de l'éq. (1.4). Dans l'éq. (1.5) ci-dessus, la grandeur $\boldsymbol{q}$ note la différence $\boldsymbol{k} \boldsymbol{n}-\boldsymbol{k}$ et est appelée le vecteur d'onde de diffusion, $h(\boldsymbol{x}, t)$ décrit la distribution spatio-temporelle des hétérogénéités au sein du milieu diffusant et l'intégrale porte sur le volume d'échantillon $V_{\text {irr }}$ irradié par le rayonnement incident.

D'après la forme même de l'éq. (1.5), c'est donc par l'intermédiaire d'une transformée de Fourier, à l'échelle définie par le vecteur d'onde de diffusion $\boldsymbol{q}$, que le champ diffusée contient les informations relatives à la distribution des hétérogénéités de l'échantillon, informations véhiculées par la fonction $h(\boldsymbol{x}, t)$. La nature physique du rayonnement utilisé n'intervient que dans l'expression détaillée de cette dernière fonction, comme indiqué ci-dessous :

Lumière : quelques complications ayant trait à la polarisation de la lumière étant omises, la fonction d'hétérogénéités « optiques » s'exprime

$$
h(\boldsymbol{x}, t)=\frac{\omega^{2}}{4 \pi c^{2}} \delta \epsilon_{r}(\boldsymbol{x}, t)
$$

où $\delta \epsilon_{r}(\boldsymbol{x}, t)$ est la fluctuation locale de constante diélectrique de l'échantillon;

Rayons X : là encore sans prendre en compte la polarisation éventuelle des rayons X ni le phénomène de diffusion anomale, la fonction d'hétérogénéités «électroniques »s'exprime

$$
h(\boldsymbol{x}, t)=r_{e} \rho_{e}(\boldsymbol{x}, t)
$$

où $r_{e} \equiv e^{2} /\left(4 \pi \epsilon_{0} m c^{2}\right)$, construit à partir des valeurs de la charge électrique $e$ et de la masse $m$ de l'électron, ainsi que de la permittivité diélectrique du vide $\epsilon_{0}$, est une longueur dont l'ordre de 
Tableau 2. Quelques longueurs de diffusion cohérente [1]. La dépendance en masse atomique $A$ est remarquable dans le cas de l'hydrogène, ainsi que pour d'autres éléments. Elle est pratiquement négligeable pour le carbone et l'oxygène.

\begin{tabular}{c||c|c|c|c} 
& ${ }^{1} \mathrm{H}$ & ${ }^{2} \mathrm{H}$ & $\mathrm{C}$ & $\mathrm{O}$ \\
\hline \hline$b_{n}[\mathrm{fm}]$ & $-3,7$ & 6,7 & 6,6 & 5,8
\end{tabular}

grandeur est $310^{-15} \mathrm{~m}$. Le deuxième symbole, $\rho_{e}(\boldsymbol{x}, t)$, note le nombre d'électrons par unité de volume au voisinage du point $\boldsymbol{x}$ et à l'instant $t-\mathrm{c}$ 'est la densité électronique ;

Neutrons : les complications liées à la polarisation du rayonnement électromagnétique trouvent leur équivalent dans le moment cinétique intrinsèque, ou spin, du neutron. Bien qu'elles soient importantes pour appréhender correctement les phénomènes de diffusion magnétique et de diffusion «incohérente», ces complications sont ignorées dans l'expression suivante de la fonction d'hétérogénéités «nucléaires »:

$$
h(\boldsymbol{x}, t)=\rho_{n}(\boldsymbol{x}, t)
$$

où $\rho_{n}(\boldsymbol{x}, t)$, la densité de longueur de diffusion neutronique, est la moyenne du nombre de noyaux atomiques d'un type isotopique donné, par unité de volume, pondéré par une longueur caractéristique de l'interaction neutron - noyau dite «longueur de diffusion cohérente ». Cette longueur joue dans le cas de la diffusion neutronique le rôle de $r_{e}$ en diffusion des rayons $\mathrm{X}$ et prend des valeurs numériques du même ordre de grandeur. Le tableau 2 montre que son signe n'est pas nécessairement positif et que sa valeur peut également être fort différente pour des isotopes distincts d'un même élément - une particularité qui est l'un des avantages spécifiques de la diffusion des neutrons.

L'échelle caractéristique à laquelle l'hétérogénéité de l'échantillon est sondée par le rayonnement est définie par le module $q$ du vecteur d'onde de diffusion et vaut, typiquement, $1 / q$. Puisque $\boldsymbol{q}=k \boldsymbol{n}-\boldsymbol{k}$ avec les notations de la Fig. 1, il vient

$$
q=2 k \sin \frac{\theta}{2}
$$

Cette relation est très souvent exprimée en fonction de la longueur d'onde $\lambda_{0}$ qu'aurait le rayonnement incident s'il se propageait à la pulsation $\omega$ dans le vide plutôt que dans l'echantillon. En utilisant les relations de dispersion, éq. (1.2) ou éq. (1.3), et leur conséquence que, dans le cas des rayons $\mathrm{X}$ comme dans celui des neutrons, la longueur d'onde du rayonnement dans l'échantillon est pratiquement identique à $\lambda_{0}$ il vient

$$
q=\frac{4 \pi n}{\lambda_{0}} \sin \frac{\theta}{2}
$$

dans le cas de lumière se propageant dans un milieu d'indice $n$ et

$$
q=\frac{4 \pi}{\lambda_{0}} \sin \frac{\theta}{2}
$$

dans le cas de rayons $\mathrm{X}$ ou de neutrons. Les gammes typiques de variation de ce paramètre $q$, compte tenu des performances instrumentales standard, sont données dans le tableau 3. Elles peuvent être étendues, du point de vue des limites inférieures, en ayant recours à des instruments spécialement conçus à cet effet, comme par exemple le spectromètre TPA du laboratoire Léon-Brillouin [2].

\subsection{Hypothèse d'incompressibilité, notion de contraste}

La forme du champ diffusé donnée par l’éq. (1.5), complétée par les liens appropriés en fonction de la nature du rayonnement entre la fonction $h$ et les sources physiques d'hétérogénéités, éq. (1.6) à 
Tableau 3. Gammes de variation du vecteur d'onde de diffusion $q$, en $\mathrm{nm}^{-1}$, selon la nature du rayonnement.

\begin{tabular}{c|c|c} 
Lumière & Rayons X & Neutrons \\
\hline \hline $210^{-3}-210^{-2}$ & $10^{-1}-10^{2}$ & $410^{-2}-210^{1}$
\end{tabular}

éq. (1.8), est très générale et valable aussi bien pour la diffusion de Bragg que pour la diffusion aux petits angles. La spécificité de cette dernière vient de la façon dont sont décrites les hétérogénéités. Ainsi, pour connaître la densité de longueur de diffusion $\rho_{n}(\boldsymbol{x}, t)$, il faut en principe identifier tous les noyaux présents à l'instant $t$ au sein d'un petit volume $\delta V$ englobant le point $\boldsymbol{x}$ et écrire

$$
\rho_{n}(\boldsymbol{x}, t)=\frac{\sum_{n} b_{n}}{\delta V}
$$

Dans cette expression, la somme ne porte que sur les noyaux effectivement présents et $b_{n}$ est la longueur de diffusion cohérente du noyau $n$. Mais quel est donc le volume $\delta V$ ? Il convient de le choisir en adéquation avec l'échelle sondée par le rayonnement, de l'ordre de $1 / q$ mais il est également indiqué de tenir compte des motivations de l'expérimentateur, comme cela apparaîtra par la suite. En diffusion aux petits angles - conventionnellement, pour $q$ plus petit que typiquement $5 \mathrm{~nm}^{-1}-$, le petit volume $\delta V$ est tel qu'il contienne déjà plusieurs molécules. Pour évaluer le terme $\sum_{n} b_{n}$ dans l'éq. (1.12), il est donc loisible, dans une $1^{\text {re }}$ étape, de regrouper tous les noyaux d'une molécule d'un type (isotopique) donné pour obtenir une longueur de diffusion propre à chaque molécule $(b)$ puis, dans une $2^{\mathrm{e}}$ étape, de compter les molécules. Pour un corps pur (du point de vue isotopique) de volume moléculaire $v$, il y a $\delta N \equiv \delta V / v$ molécules dans le petit volume $\delta V$ et l'on obtient ainsi $\rho_{n}(\boldsymbol{x}, t)=b / v$. Plus généralement, pour le mélange d'un solvant (isotopiquement pur) de volume moléculaire $v_{S}$ et de divers solutés de volumes moléculaires respectifs $v_{l}$ présents à la fraction volumique $\phi_{l}$ dans le mélange, il vient

$$
\rho_{n}(\boldsymbol{x}, t)=\sum_{l} b_{l} \frac{\phi_{l}}{v_{l}}+b_{S} \frac{1-\sum_{l} \phi_{l}}{v_{S}}
$$

puisque le petit volume $\delta V$ contient $\delta N_{l} \equiv \delta V \phi_{l} / v_{l}$ molécules de type $l$ (et $\delta N_{S} \equiv \delta V \phi_{S} / v_{S}$ molécules de solvant, avec $\left.\phi_{S}=1-\sum_{l} \phi_{l}\right)$, chacune de longueur de diffusion $b_{l}$ ( $b_{S}$ dans le cas du solvant).

Une forme équivalente est obtenue en regroupant différemment les termes de l'éq. (1.13), à savoir

$$
\rho_{n}(\boldsymbol{x}, t)=\sum_{l} \phi_{l}\left(\frac{b_{l}}{v_{l}}-\frac{b_{S}}{v_{S}}\right)+\frac{b_{S}}{v_{S}}
$$

ce qui rend très explicite le fait que, s'il est légitime de considérer le système comme incompressible le contexte étant celui de la matière molle, on entend par là que le volume moléculaire de chacun des constituants est constant -, ce sont les variations locales et instantanées de composition, décrites par les fractions volumiques $\phi_{l}(\boldsymbol{x}, t)$, qui sont à l'origine des variations locales et instantanées de densité de longueur de diffusion.

Ce modèle pour $\rho_{n}$, typique de la diffusion aux petits angles, implique qu'un corps pur ne diffuse pas les neutrons (ni les rayons X ou la lumière, d'ailleurs, vide infra) car l'éq. (1.5) conduit, pratiquement, à $\psi_{d}=0$ si $h \equiv \rho_{n}$ est indépendant de la position $\boldsymbol{x}$ au sein du volume irradié. Cette prédiction est, bien évidemment, mise en défaut si le corps pur est sensiblement compressible (au voisinage du point critique de séparation liquide - gaz, par exemple) mais également «aux grands angles », quand le petit volume $\delta V$ à considérer dans l'éq. (1.12) devient inférieur à un volume moléculaire.

Une situation de grand intérêt en diffusion des neutrons aux petits angles est celle où le «solvant» utilisé est en fait un mélange de deux espèces distinctes par leur seule composition isotopique, l'exemple le plus courant étant celui du mélange eau lourde $\left(\mathrm{D}_{2} \mathrm{O}\right)$ - eau légère $\left(\mathrm{H}_{2} \mathrm{O}\right)$, avec une fraction volumique 
$\chi$ d'eau lourde dans le «solvant». Dans ce cas, l'éq. (1.13) peut se mettre sous la forme

$$
\rho_{n}(\boldsymbol{x}, t)=\sum_{l} b_{l} \frac{\phi_{l}}{v_{l}}+\left[1-\sum_{l} \phi_{l}\right]\left[\chi \frac{b_{D}}{v_{D}}+(1-\chi) \frac{b_{H}}{v_{H}}\right]
$$

l'indice $l$ ne décrivant plus ici que les solutés au sens strict. En réarrangeant différemment les termes, il vient

$$
\rho_{n}(\boldsymbol{x}, t)=\sum_{l} \phi_{l}\left\{\frac{b_{l}}{v_{l}}-\left[\chi \frac{b_{D}}{v_{D}}+(1-\chi) \frac{b_{H}}{v_{H}}\right]\right\}+\left[\chi \frac{b_{D}}{v_{D}}+(1-\chi) \frac{b_{H}}{v_{H}}\right]
$$

ce qui fait apparaître, par comparaison avec l'éq. (1.14), la combinaison $\chi\left(b_{D} / v_{D}\right)+(1-\chi)\left(b_{H} / v_{H}\right)$ comme l'analogue de la densité de longueur de diffusion $b_{S} / v_{S}$ mais pour un «solvant» effectif de composition $\chi$. Comme noté plus haut, dans l'hypothèse d'un système incompressible, ce sont les fluctuations de composition qui sont à l'origine du signal diffusé. Aux petits angles et dans le contexte des milieux colloïdaux dispersés, la contribution des fluctuations de composition du « solvant»décrites par la valeur locale et instantanée $\chi(\boldsymbol{x}, t)$ - prend une forme très différente de celles des fluctuations de composition des solutés au sens strict - valeurs $\phi_{l}(\boldsymbol{x}, t)$-, ainsi que cela sera établi de façon quantitative dans les sections suivantes. Cela justifie l'approximation, fort usuelle, qui consiste à négliger entièrement les fluctuations de composition du « solvant », et donc à considérer $\chi$ comme une constante.

Il est maintenant temps d'introduire la notion essentielle de contraste en diffusion aux petits angles. L'éq. (1.14), convenablement généralisée si nécessaire sous la forme de l'éq. (1.16) dans le cas d'un «solvant» moyen, mélange d'espèces différant par leur composition isotopique, implique que le signal diffusé dépende du produit de $b_{l} / v_{l}-b_{S} / v_{S}$ par l'amplitude de la fluctuation de composition $\phi_{l}$ du $1^{\mathrm{e}}$ soluté. C'est la différence $\mathcal{C}_{l} \equiv b_{l} / v_{l}-b_{S} / v_{S}$ que l'on appelle alors le contraste du le soluté.

Un raisonnement très similaire permet d'identifier la différence $r_{e}\left(Z_{l}^{e} / v_{l}-Z_{S}^{e} / v_{S}\right)$ - où $Z_{l}^{e}$ (respectivement : $Z_{S}^{e}$ ) est le nombre total d'électrons de la molécule de soluté de type $l$ (resp. de solvant) - comme le terme de contraste dans l'expression de la fonction d'hétérogénéités «électroniques », appropriée pour la diffusion des rayons $\mathrm{X}$ :

$$
h(\boldsymbol{x}, t)=r_{e} \frac{Z_{S}^{e}}{v_{S}}+r_{e} \sum_{l}\left(\frac{Z_{l}^{e}}{v_{l}}-\frac{Z_{S}^{e}}{v_{S}}\right) \phi_{l}(\boldsymbol{x}, t) .
$$

Le cas de la diffusion Rayleigh est en définitive à peine différent: il s'agit toujours de diffusion «aux petits angles» puisque l'échelle définie par $1 / q$ excède largement les dimensions moléculaires, cf. Table 3. Dans le cas d'un système incompressible, les fluctuations locales de constante diélectrique à considérer d'après l'éq. (1.6) ont pour origine les fluctuations locales de composition et l'on peut donc écrire

$$
\delta \epsilon_{r}(\boldsymbol{x}, t)=\sum_{l} \frac{\partial \epsilon_{r}}{\partial \bar{\phi}_{l}} \delta \phi(\boldsymbol{x}, t)
$$

en référence à une équation d'état pour la constante diélectrique

$$
\epsilon_{r}=\epsilon_{r}\left(\phi_{1}, \phi_{2}, \cdots\right)
$$

et compte tenu du fait que la composition locale et instantanée en soluté de type $l, \phi_{l}(\boldsymbol{x}, t)$, peut s'écrire en fonction de la composition globale $\bar{\phi}_{l}$ et de la fluctuation de composition $\delta \phi_{l}(\boldsymbol{x}, t)$ sous la forme $\phi_{l}(\boldsymbol{x}, t)=\bar{\phi}_{l}+\delta \phi_{l}(\boldsymbol{x}, t)$. Il en résulte l'expression de la fonction d'hétérogénéités «optiques »

$$
h(\boldsymbol{x}, t)=\frac{\omega^{2}}{4 \pi c^{2}} \sum_{l} \frac{\partial \epsilon_{r}}{\partial \bar{\phi}_{l}} \delta \phi_{l}(\boldsymbol{x}, t)
$$


C'est donc le terme $\partial \epsilon_{r} / \partial \bar{\phi}_{l} \times \omega^{2} /\left(4 \pi c^{2}\right)$ qui joue le rôle de contraste en diffusion de la lumière ${ }^{1}$ pour le soluté de type $l$.

Il a déjà été noté à propos du corps pur incompressible qu'une fonction d'hétérogénéités constante ne donnait lieu à aucun signal en diffusion aux petits angles. En exprimant dans les éq. (1.14) ou (1.17) les $\phi_{l}(\boldsymbol{x}, t)$ comme $\bar{\phi}_{l}+\delta \phi_{l}(\boldsymbol{x}, t)$, il apparaît finalement que les diffusions Rayleigh, Thomson ou des neutrons contiennent en principe une même information, liée aux fluctuations de composition $\delta \phi_{l}(\boldsymbol{x}, t)$. Cette information est extraite de façon plus ou moins efficace en fonction du contraste propre à chaque type de rayonnement, et à une échelle spatiale fixée par le module du vecteur d'onde de diffusion $\boldsymbol{q}$.

Le champ diffusé $\psi_{d}$ n'est cependant pas directement mesurable et les détecteurs utilisés en diffusion du rayonnement ne donnent accès qu'à son intensité. De surcroît, cette mesure, même si elle peut parfois être «résolue en temps» en diffusion de la lumière ou en diffusion des rayons X (avec une source synchrotron), est le plus souvent faite sur une durée nettement supérieure aux échelles de temps caractéristique auxquelles évoluent les fluctuations $\delta \phi_{l}(\boldsymbol{x}, t)$. Le signal mesuré est donc, en définitive, une moyenne temporelle, en général assimilée à une moyenne d'ensemble à l'équilibre thermodynamique, et s'exprime comme

$$
I_{d} \propto\left\langle\left|\int_{V_{\mathrm{irr}}} d^{3} x \exp [-i \boldsymbol{q} \cdot \boldsymbol{x}] \sum_{l} \mathcal{C}_{l} \delta \phi_{l}(\boldsymbol{x}, t)\right|^{2}\right\rangle
$$

La constante de proportionnalité contient l'intensité $I_{0} \equiv\left|\psi_{0}\right|^{2}$ de l'onde plane incidente, ainsi que le facteur $1 / R^{2}$ de «dilution» de la puissance véhiculée par l'onde sphérique divergente à une (grande) distance $R$ du volume irradié - cf. éq. (1.5). Si l'on normalise par ces deux facteurs, le signe = remplace $\propto$ dans l'éq. (1.21) et l'on obtient alors une grandeur connue sous le nom de «section efficace de diffusion », homogène à une aire comme le suggère son nom puisque les contrastes $\mathcal{C}_{l}$ sont homogènes à des longueurs par unité de volume.

\section{DESCRIPTION ASYMPTOTIQUE - LOI DE POROD}

L'approche présentée ici est appropriée pour les cas où le vecteur d'onde $q$ est suffisamment grand devant l'inverse $1 / \delta$ d'une dimension structurale caractéristique des constituants du système étudié, $q \delta \gg 1$, tout en restant bien entendu dans le domaine de la diffusion aux petits angles, soit typiquement $q \leq 5 \mathrm{~nm}^{-1}$. Pour ne pas être contradictoires, ces exigences impliquent que $\delta$ soit grand par rapport aux échelles moléculaires : le système étudié est donc un milieu colloïdal. À de telles échelles spatiales, relativement locales sans atteindre néanmoins les échelles moléculaires, de nombreuses structures collö̈dales (micelles, gouttelettes d'émulsion, particules organiques ou minérales denses de forme quelconque mais pas les structures formées par les polymères en bon solvant ou en solvant $\Theta$, voir pour cela la Section 4.4) peuvent être vues comme une collection d'interfaces planes et d'orientations aléatoires $^{2}$, ces interfaces séparant deux milieux homogènes mais distincts par leur contraste. Dans la situation la plus simple, toutes les interfaces sont de même nature et séparent un milieu homogène 1 (le solvant, mettons), de densité de longueur de diffusion $b_{1} / v_{1}$, d'un milieu homogène 2 (le milieu dispersé), de densité de longueur de diffusion $b_{2} / v_{2}$. L'évaluation dans cette limite de l'éq. (1.21) donne la section efficace de diffusion selon la loi de Porod [3]:

$$
I_{d}(q)=\frac{2 \pi}{q^{4}} \times \mathcal{C}^{2} \mathcal{S}
$$

\footnotetext{
${ }^{1}$ La présence du terme en $\omega^{2}$ implique que, toutes choses égales par ailleurs, la lumière est d'autant plus efficacement diffusée que sa fréquence est plus élevée. Les couleurs bleue du ciel diurne ou rouge du soleil couchant en résultent.

2 Le cas des phases colloïdales anisotropes et orientées nécessite bien sûr un traitement spécifique, vide infra Section 5.
} 
où $\mathcal{C} \equiv b_{2} / v_{2}-b_{1} / v_{1}$ est le contraste du milieu dispersé par rapport au solvant et $\mathcal{S}$ est $l$ 'aire totale des interfaces séparant les milieux 1 et 2 dans le volume irradié $V_{\text {irr }}$.

Le facteur en $1 / q^{4}$ caractéristique de la loi de Porod a pour origine un terme en $1 / q_{z}^{2}$, module de la transformée de Fourier de la discontinuité de densité de longueur de diffusion au travers de l'interface, de normale selon l'axe $z$, qui devient $1 / q^{2} \times 1 / q^{2}$ lorsqu'est évaluée sa valeur moyenne pour toutes les orientations de ladite normale, cf. Réf. [3].

Cette expression est au demeurant facilement généralisable au cas où les interfaces de saut de densité de longueur de diffusion séparent plus de deux milieux homogènes, en remplaçant $\mathcal{C}^{2} \mathcal{S}$ par une somme des $\mathcal{C}^{2}$ associés à chaque famille de la collection d'interfaces, pondérés par les aires $\mathcal{S}$ correspondantes. La décroissance du signal reste alors en $q^{-4}$, signature caractéristique de la présence d'interfaces nettes dans les systèmes colloïdaux.

\section{DESCRIPTION THERMODYNAMIQUE}

Cette approche est utile dans la limite opposée à celle considérée Section 2, à savoir dans la limite des petits vecteurs d'onde ou, plus précisément, quand $q L$ est suffisamment petit devant $1, L$ désignant la plus grande des dimensions structurales caractéristiques. Cependant, elle n'est ni spécifique des techniques de diffusion des neutrons (la description thermodynamique est très couramment utilisée en diffusion de la lumière) ni des milieux dispersés colloïdaux.

Le point de départ reste l'éq. (1.21), réécrite sous la forme équivalente

$$
I_{d}(\boldsymbol{q})=V_{\mathrm{irr}} \int d^{3} x e^{-i \boldsymbol{q} \cdot \boldsymbol{x}} \sum_{l, m} \mathcal{C}_{l} \mathcal{C}_{m}\left\langle\delta \phi_{l}(\boldsymbol{x}) \delta \phi_{m}(\mathbf{0})\right\rangle,
$$

la thermodynamique statistique et le théorème de l'équipartition de l'énergie permettent l'évaluation explicite des fonctions de corrélation des fluctuations de composition $\left\langle\delta \phi_{l}(\boldsymbol{x}) \delta \phi_{m}(\mathbf{0})\right\rangle$, au cœur de l'éq. (3.1). Il est alors possible de calculer, en particulier dans la limite où $q \rightarrow 0$, l'intensité diffusée avec pour résultat

$$
I_{d}(\boldsymbol{q} \rightarrow \mathbf{0})=V_{\mathrm{irr}} \times k_{B} T \sum_{l, m} \mathcal{C}_{l} \mathcal{C}_{m}\left[\frac{\partial^{2} f}{\partial \phi_{l} \partial \phi_{m}}\right]^{-1}
$$

où $f$ est la densité volumique d'énergie libre du système considéré.

Dans le cas d'un système binaire, du fait que la pression osmotique $\Pi$ exercée par le mélange mis au contact, via une membrane semi-perméable, avec du solvant pur peut être exprimée sous la forme $\Pi=\phi \partial f / \partial \phi-f$, la relation entre propriétés thermodynamiques et intensité diffusée est souvent mise sous la forme équivalente

$$
I_{d}(\boldsymbol{q} \rightarrow \mathbf{0})=V_{\mathrm{irr}} \times \mathcal{C}^{2} k_{B} T \phi\left(\frac{\partial \Pi}{\partial \phi}\right)^{-1}
$$

faisant donc intervenir la compressibilité osmotique du milieu dispersé.

Une interprétation qualitative de ce résultat peut être donnée, conformément aux idées présentées Section 1.1, en identifiant le terme $k_{B} T$ lié à l'agitation thermique comme le terme à l'origine des fluctuations de concentration, le terme de compressibilité osmotique $\phi /(\partial \Pi / \partial \phi)$ agissant au contraire pour rétablir l'homogénéité locale du système.

Il est intéressant de noter que les arguments de thermodynamique statistique qui conduisent à l'éq. (3.2) peuvent être étendus à une description plus locale de la fonction de corrélation comme, par exemple, celle donnée par Ornstein et Zernike il y a près d'un siècle [4] ou encore dans le cadre d'un développement de Ginzburg-Landau de la densité volumique d'énergie libre [5]. Sous leur forme la plus simple, ces arguments font intervenir, au côté de la compressibilité osmotique, un paramètre physique 
supplémentaire $\xi$ appelé la «longueur de corrélation». L'expression de l'intensité diffusée devient alors

$$
I_{d}=V_{\mathrm{irr}} \times \mathcal{C}^{2} k_{B} T \phi \frac{(\partial \Pi / \partial \phi)^{-1}}{1+q^{2} \xi^{2}} .
$$

À l'approche d'un point critique de séparation de phases, la compressibilité osmotique et la longueur de corrélation s'accroissent indéfiniment. L'éq. (3.4) peut être utilisée pour interpréter le phénomène d'opalescence critique que l'on observe alors, surtout en diffusion de la lumière.

\subsection{Milieu dispersé idéal}

Une application élémentaire du résultat précédent au cas d'une solution (binaire) diluée repose sur le modèle des solutions idéales. La densité volumique d'énergie libre d'excès est alors donnée par la seule contribution émanant de l'entropie de mélange

$$
f_{\mathrm{exc}}=\frac{k_{B} T}{v}[\phi \ln \phi-(1-\phi) \ln (1-\phi)]
$$

où $v$ représente un volume moléculaire. Dans ce cas, l’éq. (3.3) conduit immédiatement à

$$
I_{d}(\boldsymbol{q} \rightarrow 0)=V_{\mathrm{irr}} \times \mathcal{C}^{2} v \phi .
$$

Dans ce modèle, qui ne peut bien entendu être valable que si la solution reste assez diluée pour pouvoir être considérée comme idéale, l'intensité diffusée croît en proportion directe de la concentration en soluté.

\section{2 Écarts à l'idéalité}

Lorsque la fraction en soluté augmente, le système ne peut plus être considéré comme un système idéal. Une approche perturbative reste envisageable tant que la fraction $\phi$ reste modérée, cf. Section 3.2.1 ciaprès mais d'autres méthodes sont requises au-delà, notamment en régime semi-dilué pour les solutions de polymères : cela sera l'objet de la Section 3.2.2.

\subsubsection{Correction du viriel}

Dans cette approche, on convient d'exprimer l'équation d'état pour la pression osmotique sous la forme d'un développement en puissances de $\phi$, le terme d'ordre le plus bas correspondant à la loi de van 't Hoff découlant du modèle des solutions idéales. Ainsi,

$$
\Pi=\frac{k_{B} T}{v}\left(\phi+A_{2} \phi^{2}+\cdots\right)
$$

le coefficient $A_{2}$, décrivant la $1^{\text {re }}$ correction à l'idéalité, étant appelé le $2^{\mathrm{e}}$ (pour terme du deuxième ordre) «coefficient du viriel». Il vient alors pour l'intensité diffusée

$$
I_{d}(\boldsymbol{q} \rightarrow 0)=V_{\mathrm{irr}} \times \mathcal{C}^{2} v \phi\left(1-2 A_{2} \phi+\cdots\right)
$$

une expression qui implique que, en cas d'une bonne affinité du soluté pour le solvant où $A_{2}$ est donc positif, il y a un signal diffusé moindre que dans le cas idéal. Cela est encore une fois compatible avec les idées de la Section 1.1 : la bonne affinité soluté - solvant n'est pas favorable aux grandes fluctuations locales de concentration.

Le cas des solutions diluées de polymères linéaires flexibles dans le modèle de Flory et Huggins [6] s'apparente à celui qui vient d'être traité. Ce modèle prend en compte une entropie de mélange, amoindrie par un facteur $1 / N$ caractéristique de la nature macromoléculaire des chaînes ( $N$ est le degré de polymérisation), ainsi que la plus ou moins grande affinité des monomères pour le solvant, décrite par le paramètre d'interaction $\chi$. Si $v$ note le volume moléculaire d'un monomère, l'énergie libre d'excès a 


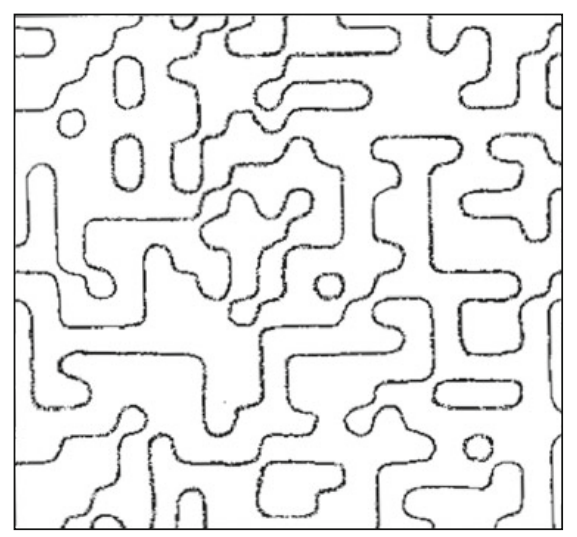

Figure 2. Représentation schématique de la structure bicontinue d'une phase «éponge » faisant apparaitre une taille caractéristique $\xi$. Une telle structure localement lamellaire se dilate quand la fraction en tensioactif diminue selon la loi de dilution $\xi \approx 1.5 \delta / \phi[8]$.

pour forme

$$
f_{\text {exc }}=\frac{k_{B} T}{v}\left[\frac{\phi}{N} \ln \phi-(1-\phi) \ln (1-\phi)+\chi \phi(1-\phi)\right] .
$$

La compressibilité osmotique est aisément déduite de l'éq. (3.9) et l'intensité diffusée s'exprime alors comme

$$
I_{d}(\boldsymbol{q} \rightarrow 0)=V_{\mathrm{irr}} \times \mathcal{C}^{2} N v \phi[1-N(1-2 \chi) \phi+\cdots] .
$$

ce qui montre qu'une mesure du degré de polymérisation $N$ est possible à partir d'une mesure absolue de l'intensité diffusée, extrapolée à angle de diffusion et concentration nuls, un procédé connu sous le nom de «méthode de Zimm » mais plutôt mis en pratique en diffusion de la lumière qu'en diffusion des neutrons.

Le rôle de 2 e coefficient du viriel est ici joué par la quantité $N(1-2 \chi) / 2$, qui s'annule lorsque $\chi=1 / 2$. Dans ce cas, qui est celui d'un solvant $\Theta$, les écarts à l'idéalité ne sont pas aussi facilement visibles en diffusion du rayonnement que pour un «bon» ou un « mauvais » solvant ( $\chi$ respectivement inférieur ou supérieur à $1 / 2$ ).

\subsubsection{Lois d'échelle}

Lorsque la fraction volumique d'une solution de polymères dépasse la fraction de recouvrement $\phi^{*}$ mais également pour d'autres systèmes comme la phase «éponge» de tensioactifs en solution, dont la structure peut être vue comme un ensemble de bicouches d'orientations aléatoires à grande échelle, séparant avec une échelle caractéristique $\xi$ deux domaines, continus mais disjoints, de solvant (cf. schéma de la Fig. 2), la densité volumique d'énergie libre d'excès ne peut plus être décrite en termes perturbatifs. Dans les deux exemples évoqués, des arguments d'invariance d'échelle conduisent à des expressions du potentiel thermodynamique sous la forme de "lois d'échelle», c'est-à-dire de dépendance de $f_{\text {exc }}$ en loi de puissance en fonction de $\phi$. Pour les solutions semi-diluées de polymères en bon solvant, l'énergie libre est donnée par [7]

$$
f_{\mathrm{exc}} \propto \frac{k_{B} T}{v} \frac{\phi}{N}\left(\frac{\phi}{\phi^{*}}\right)^{5 / 4} .
$$


Elle vaut

$$
f_{\text {exc }} \propto \frac{k_{B} T}{v}\left(\phi^{3} \ln \frac{\phi}{\phi_{0}}\right)
$$

pour une phase «éponge» dont la limite de dilution est de l'ordre de $\phi_{0}$ [8]. Une conséquence remarquable de la forme des éq. (3.11) et (3.12) est que le signal diffusé diminue lorsque la concentration en polymères ou en tensioactifs augmente - une prédiction bien vérifiée par l'expérience. Cela est associé au fait que les dimensions structurales caractéristiques (taille du «blob » dans un cas, dimension des canaux de solvant dans l'autre) deviennent plus petites quand la solution est plus concentrée.

\subsubsection{Systèmes anisotropes}

L'approche thermodynamique dans le cas de systèmes anisotropes, tels que les phases lamellaires de tensioactifs et, plus généralement, les cristaux liquides lyotropes, requiert un traitement spécifique parce que les fluctuations de composition sont fondamentalement couplées aux fluctuations élastiques de ces structures organisées. Ainsi, dans le cas d'une phase lamellaire par exemple, les fluctuations $\delta \phi$ proviennent pour l'essentiel des fluctuations de déplacement des bicouches. En repérant par la variable élastique $u\left(z, \boldsymbol{x}_{\perp}\right)$ le déplacement le long de $z$, direction moyenne d'empilement des bicouches, d'une portion de bicouche située au voisinage du point de coordonnées $\left(z, x_{\perp}\right)$, les fluctuations de composition sont en effet dominées par $\delta \phi=-\phi \partial u / \partial z$. La fonction de corrélation qui intervient dans l'éq. (3.1) s'exprime donc à partir de la fonction de corrélation des fluctuations de déplacement des couches, c'està-dire in fine en termes des constantes élastiques de compression $\bar{B}$ et de courbure des couches $K$. Il en résulte pour l'intensité diffusée [9]:

$$
I_{d}(\boldsymbol{q})=V_{\mathrm{irr}} \times \mathcal{C}^{2} \frac{k_{B} T \phi^{2} q_{z}^{2}}{\bar{B} q_{z}^{2}+K q_{\perp}^{4}} .
$$

Le signal dépend dans ce cas explicitement de l'orientation du vecteur d'onde $\boldsymbol{q}$ par rapport à la direction d'empilement des bicouches. Lorsque le vecteur d'onde est contenu dans le plan des bicouches, $q_{z}=0$ et l'intensité diffusée est nulle. En revanche si le vecteur d'onde est parallèle à la direction d'empilement, $\boldsymbol{q}_{\perp}=\mathbf{0}$ et l'intensité diffusée dans cette direction, inversement proportionnelle à $\bar{B}$, est d'autant plus grande que la phase lamellaire est plus «molle», c'est-à-dire que la constante élastique $\bar{B}$ est plus petite.

Lorsque l'échantillon n'est pas orienté, il peut être légitime de considérer que toutes les orientations possibles sont réalisées de façon équiprobable parmi les multiples cristallites présentes au sein du volume irradié. La moyenne isotrope de l'éq. (3.13), qui peut d'ailleurs être calculée de façon analytique, conduit alors à l'expression [10]

$$
I_{d}(q) \approx V_{\mathrm{irr}} \times \mathcal{C}^{2} \frac{k_{B} T \phi^{2}}{\bar{B}}\left(1-\frac{\pi}{2} \sqrt{\frac{K}{\bar{B}}} q+\cdots\right)
$$

avec une correction linéaire en $q$, à comparer à la correction quadratique caractéristique du développement de Guinier, cf. plus bas Section 4.1.

\section{MODÈLES GÉOMÉTRIQUES}

À la différence de ce qui prévalait pour les méthodes développées Sections 2 et 3, le domaine de validité de l'approche maintenant présentée ne se définit pas par référence à la valeur du vecteur d'onde $q$ mais plutôt du point de vue de la concentration. On supposera en effet dans la suite que les fractions volumique $\phi_{l}$ des espèces dispersées sont «suffisamment» petites. Cela veut dire que, même si les solutés forment en solution des structures supramoléculaires (cas des tensioactifs, par exemple), ces dernières sont (en moyenne) bien séparées les unes des autres dans le volume de l'échantillon. Il sera 
alors licite de supposer que les corrélations entre les centres de masse d'objets colloïdaux distincts sont négligeables, comme le seront les corrélations entre la position et l'orientation d'un colloïde. Pour un milieu dispersé incompressible, la densité de longueur de diffusion est convenablement représentée dans ce contexte par

$$
\rho_{n}(\boldsymbol{x}, t)=\frac{b_{S}}{v_{S}}+\sum_{l=1}^{N} \mathcal{C}_{l} \mathcal{F}_{l}\left[\boldsymbol{x}-\boldsymbol{X}_{l}(t), t\right]
$$

lorsque le volume irradié contient un ensemble de $N$ collö̈des - supposés ici pour simplifier tous homogènes mais non nécessairement identiques. Dans l'éq. (4.1), les centres de masse des objets sont repérés par les positions $\boldsymbol{X}_{l}(t)$. La fonction $\mathcal{F}_{l}$, de valeur 0 ou 1 suivant que $\boldsymbol{x}-\boldsymbol{X}_{l}(t)$ se trouve à l'extérieur ou à l'intérieur du colloïde numéro $l$, caractérise donc sa forme à l'instant $t$. Dans cette description, les fluctuations de densité de longueur de diffusion sont pilotées par les fluctuations de position, de forme et d'orientation des colloïdes formant le milieu dispersé et l'expression de principe de l'intensité diffusée est alors

$$
I_{d}(\boldsymbol{q})=\left\langle\left|\sum_{l=1}^{N} \mathcal{C}_{l} \exp \left[-i \boldsymbol{q} \cdot \boldsymbol{X}_{l}(t)\right] \int_{\text {coll. } l} d^{3} u e^{-i \boldsymbol{q} \cdot \boldsymbol{u}}\right|^{2}\right\rangle
$$

où la valeur moyenne porte à la fois sur les formes et les orientations des colloïdes présents dans le volume irradié $V$ mais également sur les positions des centres de masse. Cela étant, dans la mesure où la fraction volumique reste suffisamment basse pour que soient négligées les corrélations entre centres de masse d'objets distincts, ainsi qu'entre position, forme et orientation d'un même colloïde, l'éq. (4.2) peut être considérablement simplifiée sous la forme

$$
I_{d}(q)=\sum_{l=1}^{N} \mathcal{C}_{l}^{2}\left\langle\left|\int_{\text {coll. } l} d^{3} u e^{-i \boldsymbol{q} \cdot \boldsymbol{u}}\right|^{2}\right\rangle .
$$

Pour des colloïdes tous identiques, de fraction volumique $\phi$ et formés par l'agrégation de $\mathcal{N}$ «briques » de volume élémentaire $v$, l'éq. (4.3) est usuellement exprimée plus explicitement comme

$$
I_{d}(q)=V_{\mathrm{irr}} \times \mathcal{C}^{2} \phi \mathcal{N} v \mathrm{P}(q)
$$

Ainsi apparaît le facteur de forme $\mathrm{P}(q)$, normalisé à 1 pour $q=0$, et défini par

$$
\mathrm{P}(q)=\frac{1}{(\mathcal{N} v)^{2}} \times\left\langle\left|\int d^{3} u e^{-i \boldsymbol{q} \cdot \boldsymbol{u}}\right|^{2}\right\rangle
$$

où l'intégrale porte sur le volume, de forme éventuellement fluctuante, occupé par un colloïde. Ces expressions se généralisent facilement au cas où des populations distinctes de colloïdes se trouvent dans le volume irradié - dans une situation où la polydispersité n'est pas négligeable, par exemple. Il vient en effet

$$
I_{d}(q)=V_{\mathrm{irr}} \times \sum_{l} \mathcal{C}_{l}^{2} \phi_{l} \mathcal{N}_{l} v_{l} \mathrm{P}_{l}(q)
$$

En physico-chimie des milieux dispersés, les modèles structuraux se limitent le plus souvent à quelques formes géométriques simples comme la sphère - appropriée aussi bien pour les micelles en solution diluée que pour les micro-émulsions et les émulsions dans les situations les plus courantes de contraste, notamment -, la coquille sphérique (il s'agira alors de décrire des vésicules ou peutêtre, pour des contrastes plus subtils, des micro-émulsions ou des émulsions), le bâtonnet allongé (micelles vermiculaires, mais également phases nématiques calamitiques et phases colonnaires) et la plaquette (phases «éponge», lamellaires ou nématiques discotiques). Ces applications seront traitées Section 4.2. Un exemple archétypique d'objet déformable sera traité Section 4.3, où le modèle de 
la marche aléatoire gaussienne est introduit en vue de décrire les solutions (diluées) de polymères. Le cas d'autres géométries auto-similaires (ou « fractales») sera évoqué Section 4.4. Une collection très complète de modèles géométriques peut être trouvée en consultant la Réf. [11]. Mais il convient d'introduire tout d'abord le régime de Guinier, cf. Section 4.1, du fait de la grande universalité qui se rattache au voisinage de $q=0$ dans toutes les descriptions géométriques.

\subsection{Régime de Guinier}

Le facteur de forme, défini par l'éq. (4.5), s'exprime assez simplement dans la limite $q \rightarrow 0$ c'est-à-dire, de façon plus quantitative, quand $q$ est petit devant l'inverse de la plus grande dimension structurale caractéristique $L$ de l'objet. En utilisant le développement limité

$$
\exp (-i \boldsymbol{q} \cdot \boldsymbol{x}) \approx 1-i \boldsymbol{q} \cdot \boldsymbol{x}-\frac{(\boldsymbol{q} \cdot \boldsymbol{x})^{2}}{2}+\cdots
$$

ainsi que les propriétés statistiques $\langle\boldsymbol{q} \cdot \boldsymbol{x}\rangle=0$ et $\left\langle(\boldsymbol{q} \cdot \boldsymbol{x})^{2}\right\rangle=q^{2}\left\langle x^{2}\right\rangle / 3$ pour un vecteur $\boldsymbol{x}$ dont les fluctuations sont isotropes $^{3}$ dans l'espace de dimension 3 , il vient en effet

$$
\mathrm{P}(q) \approx 1-\frac{1}{3} q^{2} R_{G}^{2}+\cdots
$$

où le rayon de giration $R_{G}$ de l'objet est défini par

$$
R_{G}^{2}=\frac{1}{2(\mathcal{N} v)^{2}} \times \int d^{3} u d^{3} v\left\langle(\boldsymbol{u}-\boldsymbol{v})^{2}\right\rangle \equiv \frac{1}{\mathcal{N} v} \times \int d^{3} u\left\langle u^{2}\right\rangle .
$$

La définition du rayon de giration fait que cette longueur est étroitement associée à la grandeur mécanique qu'est le moment d'inertie et cette propriété est d'ailleurs à l'origine même de son nom. En effet, si l'objet colloïdal (de masse totale $M$ ) est homogène du point de vue de sa masse volumique, son moment d'inertie $J$ par rapport à son centre de masse est donné par la relation $J=M R_{G}^{2}$.

Le développement de Guinier donné par l'éq. (4.7) cesse évidemment d'être valable si $q R_{G}$ prend des valeurs trop grandes. Dans le cas où il est applicable, il est souvent mis sous la forme équivalente suivante

$$
\mathrm{P}(q) \approx \exp \left(-q^{2} R_{G}^{2} / 3\right)
$$

à l'origine de la représentation dite «de Guinier» où le logarithme de l'intensité diffusée est tracé en fonction de $q^{2}$. La pente (négative) de la droite qui en résulte est proportionnelle au carré du rayon de giration (si tous les objets sont en moyenne identiques), ou à ce que l'on appelle le carré du rayon de giration apparent pour des objets présentant une dispersion de taille ou de forme - rayon apparent car cette longueur n'est alors plus une caractéristique géométrique intrinsèque mais dépend de facteurs de contraste et des fractions volumique, cf. éq. (4.6).

\subsection{Sphères et cylindres}

\subsubsection{Boules et coquilles}

Dans les cas où les objets collö̈daux d'intérêt peuvent être représentés par une forme géométrique «simple», les calculs explicites de l'intégrale, ainsi que de la valeur moyenne qui définissent le facteur de forme - éq. (4.5) - peuvent être effectués de façon exacte ou quasi-exacte. C'est notamment le cas

\footnotetext{
${ }^{3}$ L'isotropie de la distribution des orientations est essentielle mais le colloïde peut lui-même être un objet anisotrope, comme un plaquette ou un bâtonnet par exemple. Cela exclut cependant les phases nématiques ou smectiques de tels colloïdes - pour lesquelles l'approximation d'indépendance statistique des fluctuations de centre de masse et d'orientation introduite pour arriver à l'éq. (4.4) n'est de toutes les façons plus valable.
} 
avec les objets sphériques, qu'il s'agisse de sphères pleines (ou boules), ou encore de sphères creuses (ou coquilles), pour autant que la matière qui les constitue soit répartie d'une manière homogène dans le volume de l'objet. Pour des objets sphériques, la question de l'orientation - et donc celle de la valeur moyenne associée - ne se pose tout simplement pas. La valeur moyenne sur les formes possibles de l'objet n'a pas non plus de raison d'être puisque la forme, en l'occurrence, sphérique, est définie a priori.

Le calcul est élémentaire dans un système de coordonnées sphériques avec pour origine le centre de la sphère (creuse ou pleine) et pour axe polaire la direction définie par le vecteur d'onde de diffusion $\boldsymbol{q}$. Il vient

$$
\mathrm{P}_{b}(q)=9\left[\frac{\sin q R-q R \cos q R}{q^{3} R^{3}}\right]^{2}
$$

dans le cas d'une boule de rayon $R$, ainsi que

$$
\mathrm{P}_{c}(q)=9 \frac{\left[\sin q R_{>}-\sin q R_{<}-q R_{>} \cos q R_{>}+q R_{<} \cos q R_{<}\right]^{2}}{q^{6}\left[R_{>}^{3}-R_{<}^{3}\right]^{2}}
$$

dans le cas d'une coquille de rayon externe $R_{>}$et de rayon interne $R_{<}$.

Dans la limite, typiquement pertinente pour les vésicules, d'une coquille de faible épaisseur, c'està-dire lorsque $\delta \equiv R_{>}-R_{<}$est très petit devant $R_{>}$, le facteur de forme de la coquille sphérique se simplifie (pour autant que $q \delta$ reste petit devant 1) sous la forme

$$
\mathrm{P}_{c}(q) \approx \frac{\sin ^{2} q R}{q^{2} R^{2}}
$$

où $R$ représente maintenant, par exemple, $R_{>}$.

Les expressions éq. (4.10) et (4.12) peuvent être facilement développées en puissances de $q R$, ce qui permet de relier le rayon de giration de la boule ou de la coquille à la plus grande de dimensions géométriques de ces objets, c'est-à-dire au seul rayon $R$ dans les deux cas considérés. Le rayon de giration d'une coquille fine est donné, conformément à l'intuition, par la relation $R_{G}=R$. Le rayon de giration d'une boule est quant à lui donné par

$$
R_{G}=\sqrt{\frac{3}{5}} R
$$

Dans la limite, opposée, des grands vecteurs d'onde de diffusion, on retrouve la loi de Porod présentée sous sa forme générale Section 2, même si la présence des oscillations dues aux fonctions trigonométriques conduit à en nuancer la formulation. La Fig. 3 l'illustre ici pour une boule. La courbe en trait plein est la représentation de la fonction donnée par l'éq. (4.10). La courbe en pointillés représente le terme dominant aux grands vecteurs d'onde de diffusion, à savoir la fonction $9 \cos ^{2}(q R) /(q R)^{4}$, qui apparaît donc comme une approximation raisonnable du facteur de forme pour $q \geq 6 / R$, typiquement. Enfin, la droite - d'équation $9 /\left(2 q^{4} R^{4}\right)$ puisque l'aire totale des sphères dans le volume irradié est $\mathcal{S}=V_{\text {irr }} \phi \times 4 \pi R^{2} /\left(4 \pi R^{3} / 3\right)$ - est la prédiction de la loi de Porod. Cette dernière décrit donc de façon quantitative le facteur de forme pour $q \gg 1 / R$ à condition de remplacer la fonction oscillante, ici $\cos ^{2}(q R)$, par sa valeur moyenne $1 / 2$. Les oscillations sont parfois difficiles à mettre en évidence du fait des imperfections expérimentales (polydispersité et résolution finie, essentiellement) et la nuance apportée à la loi de Porod est alors de faible portée. En revanche, l'observation de telles oscillations dans la représentation dite « de Porod» où l'intensité diffusée multipliée par $q^{4}$ est tracée en fonction de $q$ donne un moyen commode - et très utilisé en pratique - pour estimer la taille $R$ de l'objet.

Si l'objet est une coquille sphérique, il y a deux - et non plus une seule - longueurs caractéristiques de sa structure. Ces deux longueurs sont, par exemple, les rayons internes et externes de la coquille, $R_{<}$ et $R_{>}$respectivement, mais il est plus fructueux de considérer la plus grande longueur caractéristique, à savoir $R_{>}$, ainsi que l'épaisseur $\delta$ de la coquille, déjà définie comme $R_{>}-R_{<}$, qui est aussi la plus 


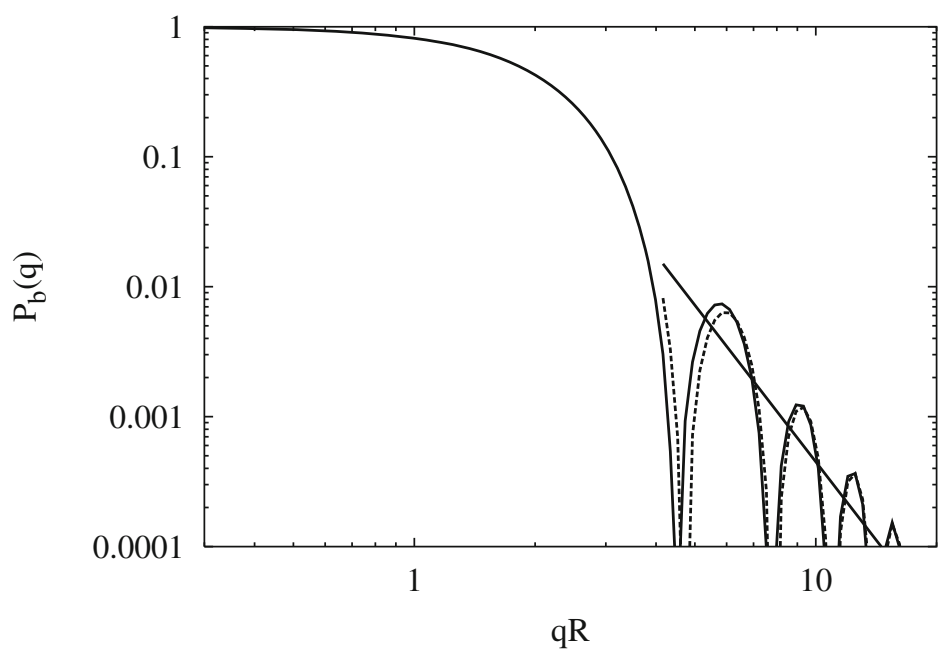

Figure 3. Facteur de forme d'une boule de rayon $R$, forme limite aux grands vecteurs d'onde de diffusion $q$ et loi de Porod.

petite longueur caractéristique lorsque la coquille est mince. Trois domaines de vecteurs d'onde de diffusion apparaissent ainsi, les deux premiers étant bien évidemment le domaine de Guinier, lorsque $q R_{>}$est petit devant 1 , et le domaine de Porod si $q \delta$ est grand devant 1 . Le $3^{\mathrm{e}}$ domaine, dit domaine intermédiaire parce que situé entre les deux domaines précédents, est ici défini par :

$$
\frac{1}{R_{>}} \ll q \ll \frac{1}{\delta}
$$

L'existence même du domaine intermédiaire est en fait une propriété générale de tous les objets dont la forme est définie par au moins deux échelles caractéristiques très différentes. D'autres exemples seront donnés par la suite.

Dans le cas considéré ici, l'approximation du facteur de forme de la coquille fine donnée par l'éq. (4.12) est en fait valable aussi bien dans le régime de Guinier que dans le domaine intermédiaire. La Fig. 4 donne une illustration de l'allure de ce résultat, ainsi que celle du facteur de forme complet de la coquille sphérique, avec une structure complexe d'oscillations aux échelles $1 / R$ et $1 / \delta$. Si les contraintes expérimentales le permettent, les oscillations à l'échelle $1 / R$ sont révélées par la représentation dite « de Kratky» où l'intensité diffusée multipliée par $q^{2}$ est tracée en fonction de $q$. Une détermination de $R$ dans le régime intermédiaire, indépendante de celle ayant recours à la mesure du rayon de giration, est alors possible. De façon similaire, la représentation de Porod est suceptible de rendre visibles les oscillations à l'échelle $1 / \delta$, ce qui donne accès à cette grandeur par une mesure dans le régime asymptotique.

\subsubsection{Bâtonnets et disques}

Ces deux objets sont en fait géométriquement identiques, puisqu'il s'agit dans les deux cas de cylindres. Pour un bâtonnet, la hauteur $L$ du cylindre est cependant supposée grande devant son diamètre $2 R$ alors que l'inégalité est inverse pour le disque, d'épaisseur $\delta$ petite devant son diamètre $2 R$. Les cylindres (longs ou plats) sont de plus supposés pouvoir fluctuer librement quant à leur orientation, définie par exemple par rapport à la direction du vecteur d'onde de diffusion $\boldsymbol{q}$, et quant à leur position. Cela requiert bien sûr que la fraction volumique des objets dans le milieu diffusant soit petite devant la fraction volumique de recouvrement $\phi^{*}$, donnée (en ordre de grandeur) par $\phi^{*} \simeq R^{2} / L^{2}$ dans le cas des bâtonnets ou par $\phi^{*} \simeq \delta / R$ dans le cas des disques. 


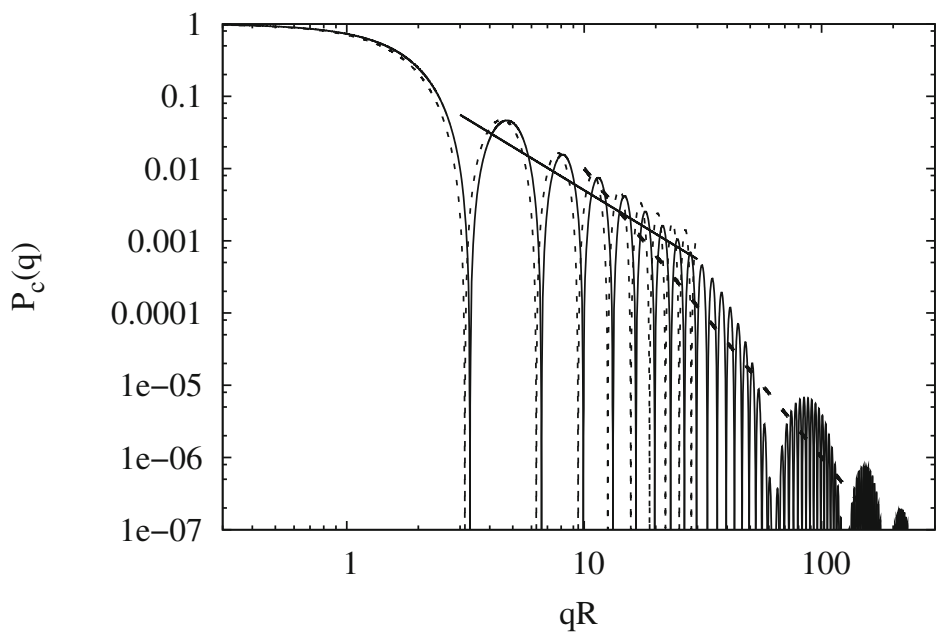

Figure 4. Facteur de forme d'une coquille de rayon externe $R$ et d'épaisseur $\delta=R / 10$, ainsi la forme approchée pour le domaine de Guinier et le domaine intermédiaire. La droite en trait plein, de pente -2 , représente $1 /\left(2 q^{2} R^{2}\right)$, celle en pointillés, de pente -4 , la limite de Porod $1 /\left(q^{4} R^{2} \delta^{2}\right)$.

Pour le calcul de l'intégrale qui définit le facteur de forme, éq. (4.5), il est commode de repérer les points de l'espace dans un système de coordonnées cylindriques dont l'axe est défini par l'orientation instantanée du cylindre. Un vecteur $\boldsymbol{x}$ a pour représentation $\boldsymbol{x}=z \boldsymbol{u}_{z}+r\left(\cos \theta \boldsymbol{u}_{x}+\sin \theta \boldsymbol{u}_{y}\right)$ et le vecteur d'onde de diffusion a pour composantes $\boldsymbol{q}=q_{z} \boldsymbol{u}_{z}+q_{\perp}\left(\cos \Theta \boldsymbol{u}_{x}+\sin \Theta \boldsymbol{u}_{y}\right)$. L'éq. (4.5) s'écrit alors (les notations sont adaptées pour des bâtonnets. Pour des plaquettes, il suffit de remplacer $L$ par $\delta$ )

$$
\mathrm{P}_{\text {bât. }}(q)=\frac{1}{\pi^{2} R^{4} L^{2}}\left\langle\left|\int_{-L / 2}^{L / 2} d z e^{i q_{z} z} \int_{0}^{R} d r r \int_{0}^{2 \pi} d \theta e^{i q_{\perp} r \cos (\theta-\Theta)}\right|^{2}\right\rangle
$$

où la valeur moyenne fait ici référence aux seules fluctuations d'orientation de l'axe des cylindres.

L'intégrale sur l'angle polaire $\theta$ dans le plan perpendiculaire à l'axe du cylindre s'exprime de façon analytique à l'aide d'une fonction de Bessel $\mathrm{J}_{0}$ (la définition mathématique et de nombreuses propriétés de cette fonction, ainsi que d'autres du même genre dites «spéciales », peuvent être trouvées dans de multiples références, par exemple [12]) sous la forme

$$
\mathrm{P}_{\text {bât. }}(q)=\frac{4}{R^{4} L^{2}}\left\langle\left|\int_{-L / 2}^{L / 2} d z e^{i q_{z} z} \int_{0}^{R} d r r \mathrm{~J}_{0}\left(q_{\perp} r\right)\right|^{2}\right\rangle .
$$

L'intégrale sur la coordonnée longitudinale $z$ est élémentaire et celle sur la coordonnée transverse fait une nouvelle fois intervenir une fonction de Bessel, d'ordre 1 cette fois

$$
\mathrm{P}_{\text {bât. }}(q)=\frac{16}{R^{2} L^{2}}\left\langle\frac{\sin ^{2}\left(q_{z} L / 2\right)}{q_{z}^{2}} \frac{\mathrm{J}_{1}^{2}\left(q_{\perp} R\right)}{q_{\perp}^{2}}\right\rangle \text {. }
$$

Pour le calcul de la valeur moyenne sur les orientations, il convient maintenant de se placer dans un système de coordonnées sphériques, mieux adapté, dont l'axe polaire est défini par la direction de $\boldsymbol{q}$. S'il est bien légitime de supposer que toutes les orientations sont équiprobables, en phase isotrope par conséquent, l'expression du facteur de forme, éq. (4.17), se transforme pour des bâtonnets en

$$
\mathrm{P}_{\text {bât. }}(q)=\int_{0}^{1} d x\left[\frac{2 \sin (q L x / 2)}{q L x}\right]^{2}\left[2 \frac{\mathrm{J}_{1}\left(q R \sqrt{1-x^{2}}\right)}{q R \sqrt{1-x^{2}}}\right]^{2}
$$


et pour des disques en

$$
\mathrm{P}_{\text {disq. }}(q)=\int_{0}^{1} d x\left[\frac{2 \sin (q \delta x / 2)}{q \delta x}\right]^{2}\left[2 \frac{\mathrm{J}_{1}\left(q R \sqrt{1-x^{2}}\right)}{q R \sqrt{1-x^{2}}}\right]^{2}
$$

puisqu'il suffit de remplacer la longueur $L$ du bâtonnet par l'épaisseur $\delta$ du disque.

Le calcul exact ne peut pas être effectué sous forme analytique dans le cas général. Dans la limite d'objets très anisotropes (avec, donc, $L \gg R$ dans le cas des bâtonnets, ou bien $R \gg \delta$ pour les disques) des approximations utiles peuvent être faites. Dans le régime de Guinier, $q$ est petit devant $1 / L$ (bâtonnets) ou $1 / R$ (disques). Le rayon de giration $R_{G}$, défini à partir du développement limité du facteur de forme, vaut $R_{G}^{2}=L^{2} / 12$ dans le cas des bâtonnets et $R_{G}^{2}=R^{2} / 2$ pour les disques. Par ailleurs, à partir du moment où l'inégalité $q L \gg 1$ (bâtonnets) ou $q R \gg 1$ (disques) se met à être vérifiée (domaine intermédiaire et limite de Porod), la fonction sinus (resp. de Bessel) varie beaucoup plus rapidement en $x$ autour de $x=0$ (resp. autour de $x=1$ ) que la fonction de Bessel (resp. sinus), et prend en outre des valeurs négligeables en dehors du voisinage immédiat de $x=0$ (resp. $x=1$ ). Il devient donc raisonnable de faire l'approximation

$$
\mathrm{P}_{\text {bât. }}(q) \approx\left[\frac{2 \mathrm{~J}_{1}(q R)}{q R}\right]^{2} \int_{0}^{1} d x\left[\frac{2 \sin (q L x / 2)}{q L x}\right]^{2} \approx\left[\frac{2 \mathrm{~J}_{1}(q R)}{q R}\right]^{2} \frac{\pi}{q L}
$$

dans le cas des bâtonnets et

$$
\begin{aligned}
\mathrm{P}_{\text {disq. }}(q) & \approx\left[\frac{2 \sin (q \delta / 2)}{q \delta}\right]^{2} \int_{0}^{1} d x\left[\frac{2 \mathrm{~J}_{1}\left(q R \sqrt{1-x^{2}}\right)}{q R \sqrt{1-x^{2}}}\right]^{2} \\
& \approx\left[\frac{2 \sin (q \delta / 2)}{q \delta}\right]^{2} \frac{2}{q^{2} R^{2}}
\end{aligned}
$$

dans le cas des disques.

Tant que l'on se trouve dans le domaine intermédiaire au sens strict, à savoir avant le régime de Porod, lorsque $1 / L \ll q \leq 1 / R$ pour des bâtonnets (resp. $1 / R \ll q \leq 1 / \delta$ pour des disques), le facteur de forme décroît de façon monotone comme $q^{-1}$ (resp. $q^{-2}$ ). Il est souvent représenté, par exponentiation du développement limité de la fonction appropriée, comme

$$
\mathrm{P}_{\text {bât. }}(q) \approx \frac{\pi}{q L} e^{-q^{2} R^{2} / 4}
$$

dans le cas des bâtonnets, ou comme

$$
\mathrm{P}_{\text {disq. }}(q) \approx \frac{2}{q^{2} R^{2}} e^{-q^{2} \delta^{2} / 12}
$$

dans le cas des disques.

Finalement, dans le domaine asymptotique où $q R \gg 1$ (resp. $q \delta \gg 1$ ), les éq. (4.20) ou (4.21) impliquent que l'intensité diffusée varie globalement comme $q^{-4}$ dans les deux cas (la forme asymptotique de la fonction de Bessel $\mathrm{J}_{1}(x)$ est en effet $\sqrt{\frac{2}{\pi x}} \cos \left(x-\frac{3 \pi}{4}\right)$ pour $\left.x \rightarrow \infty\right)$, avec des annulations du signal contrôlées par les zéros de $\cos \left(q R-\frac{3 \pi}{4}\right)$ dans le cas des bâtonnets ou par ceux de $\sin (q \delta / 2)$ avec des disques. La loi de Porod est une fois de plus retrouvée sous la forme présentée en Section 2, toujours à la nuance près qu'il faut remplacer les fonctions trigonométriques par leur valeur moyenne $1 / 2$.

La Fig. 5 donne, à partir des approximations discutées ci-dessus, une représentation graphique du facteur de forme d'un bâtonnet tel que $L=10 R$. De même, la Fig. 6 donne une représentation graphique du facteur de forme d'un disque avec $R=10 \delta$. Abstraction faite des oscillations dans le domaine intermédiaire pour le facteur de forme d'une coquille sphérique, oscillations en pratique 


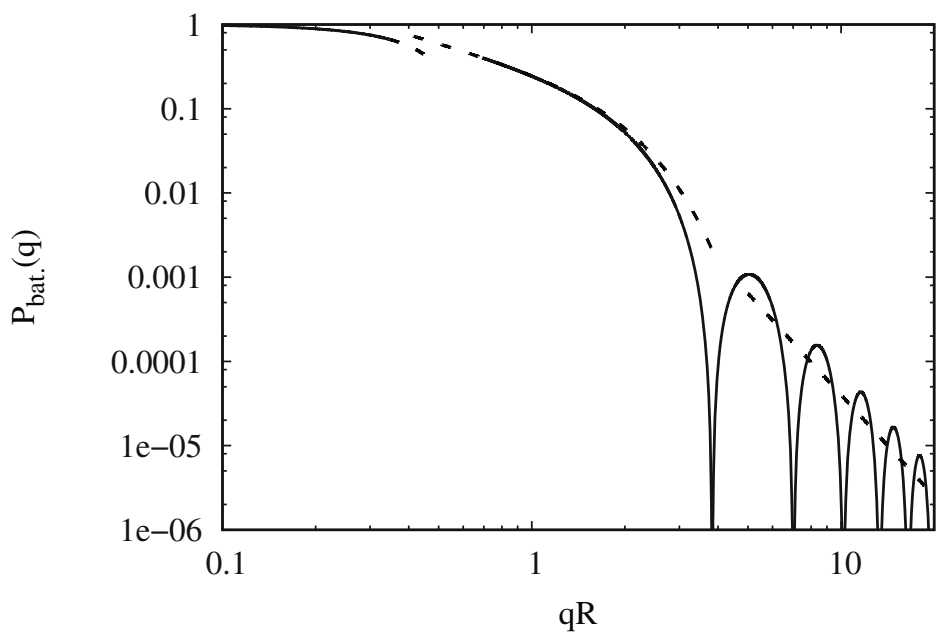

Figure 5. Facteur de forme approché d'un bâtonnet de longueur $L$ et de rayon $R=L / 10$. Les courbes en trait continu représentent la limite de Guinier, ainsi que l'éq. (4.20). Les courbes en pointillés représentent, pour des valeurs croissantes de $q R$, l'extrapolation de la loi de Guinier, l'expression (4.22) pour le régime intermédiaire et le régime de Porod en $4 /\left(q^{4} L R^{3}\right)$.

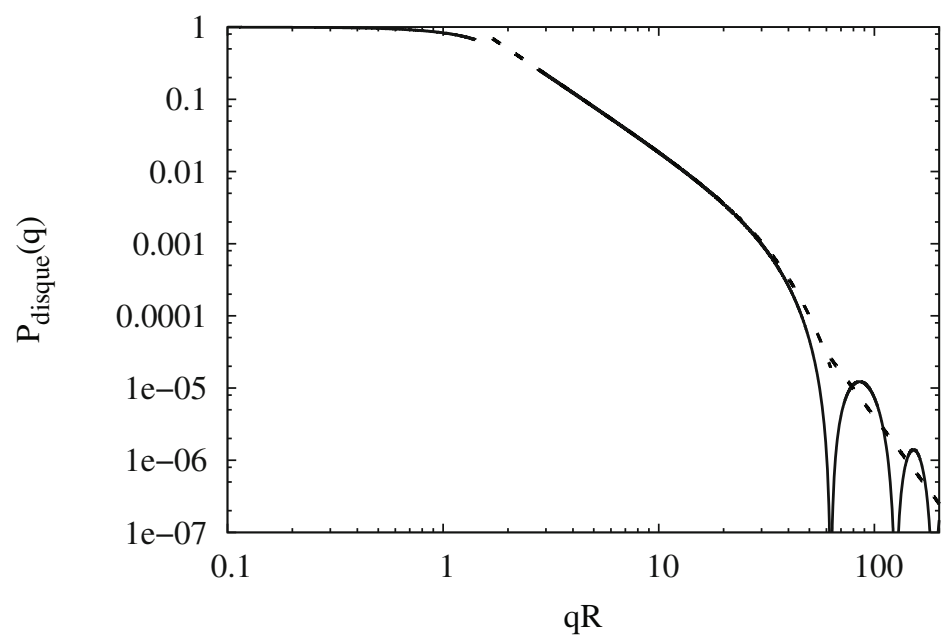

Figure 6. Facteur de forme approché d'un disque de rayon $R$ et d'épaisseur $\delta=R / 10$. Les courbes en trait continu représentent la limite de Guinier, ainsi que l'éq. (4.21). Les courbes en pointillés représentent, pour des valeurs croissantes de $q R$, l'expression (4.23) pour le régime intermédiaire et le régime de Porod en $4 /\left(q^{4} R^{2} \delta^{2}\right)$.

souvent inobservables du fait des imperfections expérimentales, une comparaison entre les Fig. 4 et 6 indique une grande similitude entre les facteurs de forme des coquilles (minces) et des disques (plats), traduction de la similitude de ces structures à une échelle suffisamment locale pour que la courbure de la coquille ne soit pas perceptible. Par ailleurs, comme c'était le cas pour les boules et les coquilles, les oscillations éventuellement révélées par une représentation de Porod de l'intensité diffusée donneront accès à l'épaisseur $\delta$ des disques ou au rayon $R$ des bâtonnets, selon que les objets cylindriques sont aplatis ou allongés. 


\subsection{Marche aléatoire gaussienne}

Les objets étudiés dans les sections précédentes - sphères ou cylindres - sont des objets implicitement considérés comme rigides: leur forme est donnée une fois pour toute. Le modèle de la marche aléatoire gaussienne, également connu sous la dénomination de "pelote statistique », permet de traiter un cas simple d'objet déformable et fournit, de surcroît, une représentation intéressante du signal diffusé par certaines solutions diluées de polymères flexibles.

Dans le modèle de la pelote statistique, l'objet est défini par un ensemble discret - plutôt que continu, comme auparavant - de $N+1$ points formant une chaîne linéaire flexible décrite de la façon suivante:

- Le premier élément de la chaîne occupe une position arbitraire dans l'espace, repérée par $\boldsymbol{R}_{0}$;

- La position relative de l'élément $l+1$ par rapport à l'élément $l$ est telle que $\boldsymbol{R}_{l+1}-\boldsymbol{R}_{l}$ soit un vecteur d'orientation aléatoire mais de module constant $a$;

- Il n'y a aucune corrélation statistique entre les orientations de $\boldsymbol{R}_{l+1}-\boldsymbol{R}_{l}$ et de $\boldsymbol{R}_{m+1}-\boldsymbol{R}_{m}$ si $l \neq m$.

La transposition au cas discret de l'équation (4.5) donne

$$
\mathrm{P}_{D}(q)=\frac{\left\langle\left|\sum_{l=0}^{N} \exp \left(-i \boldsymbol{q} \cdot \boldsymbol{R}_{l}\right)\right|^{2}\right\rangle}{(N+1)^{2}}=\frac{1}{(N+1)^{2}} \sum_{l=0}^{N} \sum_{m=0}^{N}\left\langle\exp \left[-i \boldsymbol{q} \cdot\left(\boldsymbol{R}_{l}-\boldsymbol{R}_{m}\right)\right]\right\rangle
$$

Les propriétés statistiques du modèle de la marche aléatoire permettent d'établir que

$$
\left\langle\exp \left[-i \boldsymbol{q} \cdot\left(\boldsymbol{R}_{l}-\boldsymbol{R}_{m}\right)\right]\right\rangle=\exp \left[-\frac{q^{2} a^{2}|l-m|}{6}\right]
$$

dans la limite où $|l-m| \gg 1$ car les fluctuations de $\boldsymbol{R}_{l}-\boldsymbol{R}_{m}$ sont alors régies par une statistique gaussienne. On considère usuellement que l'éq. (4.25) reste valide même lorsque les rangs $l$ et $m$ sont proches l'un de l'autre. Dans le cadre de cette hypothèse, la somme double de l'éq. (4.24) peut être calculée de façon exacte, ce qui conduit à l'expression, due à Debye [13], du facteur de forme de la pelote statistique

$$
\mathrm{P}_{D}(q)=\frac{2}{q^{4} R_{G}^{4}}\left[q^{2} R_{G}^{2}-1+\exp \left(-q^{2} R_{G}^{2}\right)\right]
$$

où le paramètre noté $R_{G}$ est effectivement le rayon de giration de l'objet. Il est au demeurant donné en fonction de la taille $N$ et du pas $a$ de la marche aléatoire par $R_{G}=N a^{2} / 6$.

Il est remarquable dans ce modèle que, s'il y a bien un régime de Guinier et un régime intermédiaire suivant que le vecteur d'onde de diffusion $q$ est petit ou grand devant $1 / R_{G}$, il n'y a pas, formellement, de régime de Porod. Cela tient à l'absence d'interfaces, inhérente à la nature même du modèle géométrique adopté. L'allure du facteur de forme de la pelote statistique, aussi connu sous le nom de «fonction de Debye», est donnée Fig. 7.

\subsection{Géométries fractales}

L'invariance d'échelle a été évoquée, Section 3.2.2, à l'occasion de l'approche thermodynamique dévolue aux solutions semi-diluées de polymères ou à la phase «éponge » des solutions de tensioactifs mais c'est d'une propriété géométrique dont il est, avant tout, question. Elle fait référence au fait qu'une structure, par exemple celle formée lors d'un processus cinétique d'agrégation, présente une « allure » statistiquement similaire sur une gamme étendue d'échelles de longueur. Elle est alors qualifiée de «fractale». Sans entrer dans les détails de la description mathématique de tels objets, il suffira ici de mentionner les trois paramètres minimaux requis en vue de leur étude par diffusion du rayonnement : leur taille globale, par exemple évaluée à l'aide du rayon de giration $R_{G}$ de l'objet, une longueur «de coupure» $a$ qui caractérise la taille des constituants élémentaires et leur dimension fractale $D_{F}$. 


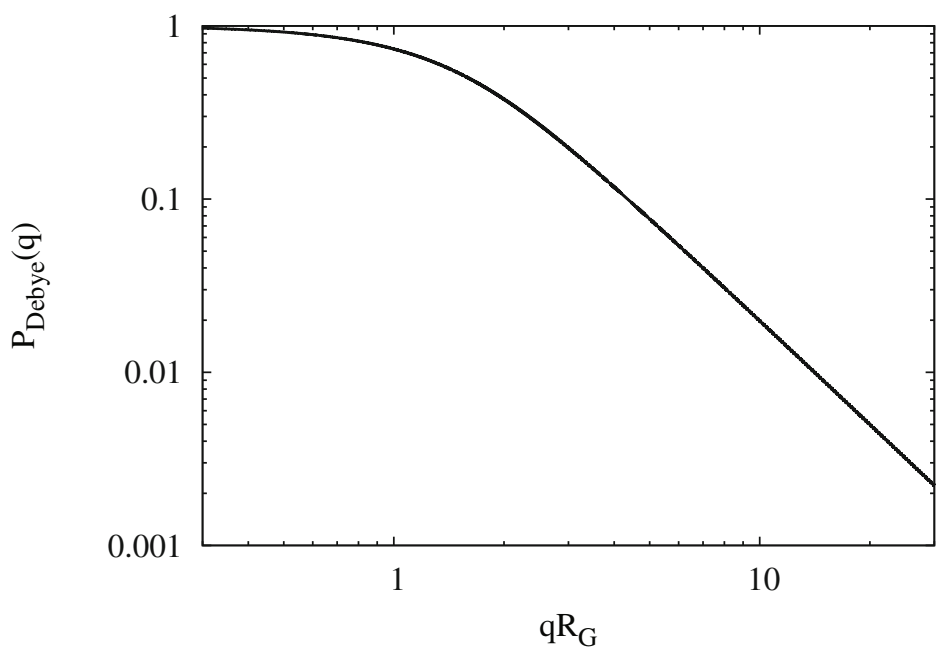

Figure 7. Facteur de forme d'une pelote statistique de rayon de giration $R_{G}$. La variation est en $2 /\left(q^{2} R_{G}^{2}\right)$ dans le régime intermédiaire. Il n'y a pas, pour ce modèle géométrique dépourvu d'interfaces, de régime de Porod.

L'auto-similarité n'est vérifiée que pour des échelles $R$ comprises entre $a$ et $R_{G}$ où, conséquence de la définition de la dimension fractale, le nombre $N(R)$ de constituants élémentaires dans une partie, quelconque mais de taille $R$, de l'objet varie en proportion de $R^{D_{F}}$.

De nombreux modèles pour l'intensité diffusée par une structure fractale ont été proposés, par exemple [14]. Ils décrivent, essentiellement, différentes interpolations entre le régime de Guinier pour $q R_{G} \lesssim 1$ et le régime intermédiaire en $1 / q^{D_{F}}$ lorsque l'échelle devient suffisamment locale, c'est-àdire $q$ suffisamment grand, pour que l'auto-similarité se vérifie. La Fig. 8 en donne deux exemples, tirés de SASfit [11]. La seule différence entre les deux modèles vient du niveau d'intensité dans le régime intermédiaire, qui résulte d'une plus ou moins grande extension de la zone de transition: il convient donc en général d'être prudent dans l'interprétation de spectres en termes de géométries fractales et il est alors souhaitable d'avoir accès à une gamme de vecteurs d'onde (et, par voie de conséquence, d'intensité !) aussi étendue que possible.

\section{STRUCTURES DENSES OU ORDONNÉES}

Une expérience de diffusion des neutrons aux petits angles est souvent conduite en dehors des régimes précédemment évoqués de vecteurs d'onde et de concentration et donc en dehors du domaine de validité des approximations faites jusqu'ici. C'est toujours le cas lorsque la structure du système étudié est cristalline, ou cristal-liquide de type smectique ou colonnaire : l'existence de l'ordre translationnel dans une, deux ou trois dimensions de l'espace impose que les positions des constituants structuraux soient corrélées. Des corrélations, d'orientation cette fois, existent dans les phases nématiques, du fait de l'existence d'une direction privilégiée. Il y a également des corrélations dans les systèmes désordonnés, isotropes à grande échelle, aux concentrations supérieures à la concentration de recouvrement qui peut justement être définie comme la concentration au-dessus de laquelle les fluctuations de position ou d'orientation cessent d'être indépendantes. Il en est de même dans le cas de dispersions de colloïdes sphériques si les interactions entre constituants ne sont pas réellement négligeables, soit parce qu'elles sont à longue portée (systèmes chargés à faible force ionique), soit parce que le système est concentré. 


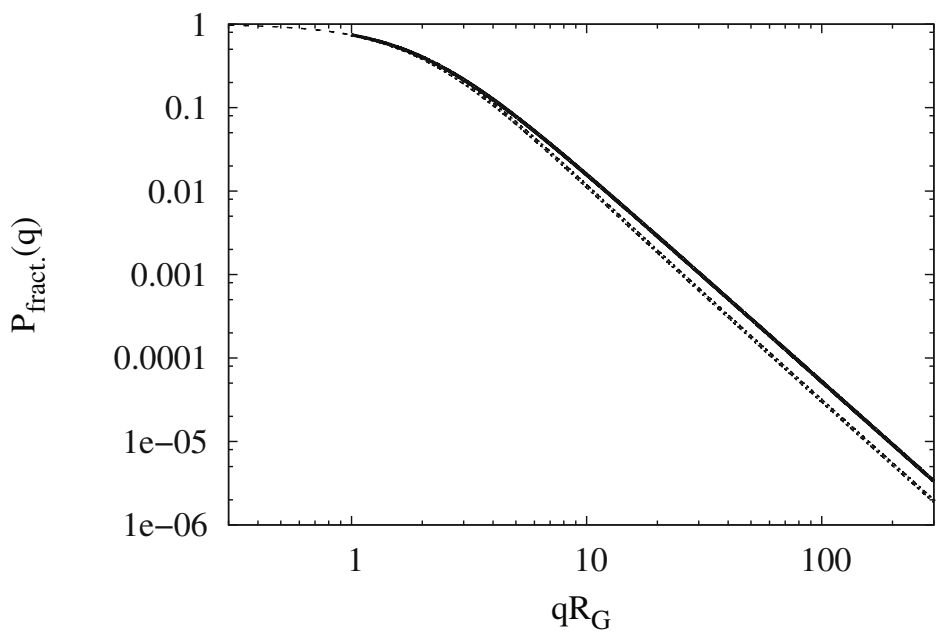

Figure 8. Facteurs de forme pour une structure de géométrie fractale caractérisée par son rayon de giration $R_{G}$ et sa dimension fractale $D_{F}$ (la valeur choisie est $D_{F}=2.5$ ). La décroissance suit une même la loi de puissance $1 / q^{D_{F}}$ dans le régime intermédiaire mais avec une intensité différente pour les deux modèles présentés - trait plein et pointillés. Ces modèles cessent d'être valables aux échelles locales où, du fait de la (petite) taille finie des constituants élémentaires de l'agrégat, l'hypothèse d'invariance d'échelle n'est plus vérifiée. Il y a donc, en principe, un régime de Porod, non décrit par les modèles, aux valeurs de $q$ les plus grandes.

La modélisation de l'intensité diffusée pose de sérieuses difficultés car la forme générale de l'éq. (4.2) de la Section 4, à savoir

$$
I_{d}(\boldsymbol{q})=\sum_{l=1}^{N} \sum_{m=1}^{N} \mathcal{C}_{l} \mathcal{C}_{m}\left\langle\exp \left\{-i \boldsymbol{q} \cdot\left[\boldsymbol{X}_{l}(t)-\boldsymbol{X}_{m}(t)\right]\right\} \int_{\text {coll. } l} d^{3} u e^{-i \boldsymbol{q} \cdot \boldsymbol{u}} \int_{\text {coll. } m} d^{3} v e^{i \boldsymbol{q} \cdot \boldsymbol{v}}\right\rangle
$$

fait intervenir un grand nombre de variables fluctuantes de position, de forme et d'orientation qui sont, a priori, corrélées les unes avec les autres.

Une approximation envisageable pour quelque peu simplifier l'éq. (5.1) revient à considérer que les constituants structuraux du système sont des colloïdes de formes et d'orientation strictement non fluctuantes, ce qui permet de ne faire porter la valeur moyenne que sur les seules variables de position $\boldsymbol{X}_{l}(t)$ des centres de masse des objets dispersés. Ce schéma est toujours acceptable pour décrire une phase, cristalline ou non, de sphères colloïdales qui ne présentent évidemment pas de fluctuations d'orientation. Il peut l'être également dans le cas de phases nématiques, smectiques ou colonnaires l'orientation non fluctuante des colloïdes anisotropes se définit alors par référence à la direction connue à l'échelle macroscopique du directeur - mais doit évidemment être rejeté dans le cas d'une suspension isotrope d'objets anisotropes, phase de micelles vermiculaires ou phase «éponge» par exemple. Dans le cadre de cette approximation, l'éq. (5.1) devient (en supposant des objets tous identiques, pour simplifier)

$$
I_{d}(\boldsymbol{q})=V_{\mathrm{irr}} \times \mathcal{C}^{2} \phi \mathcal{N} v \mathrm{P}(\boldsymbol{q}) \times \mathrm{S}(\boldsymbol{q})
$$

où le facteur de forme est défini par une expression analogue à l'éq. (4.5) de la Section 4, mais dépend maintenant en général explicitement de l'orientation du vecteur d'onde, et où le facteur de structure (parfois qualifié de facteur de structure statique du fait qu'il soit indépendant du temps pour un système à l'équilibre thermodynamique) est défini, pour un ensemble de $N$ colloïdes, par

$$
\mathrm{S}(\boldsymbol{q})=\frac{1}{N} \sum_{l, m}\left\langle\exp \left\{-i \boldsymbol{q} \cdot\left[\boldsymbol{X}_{l}(t)-\boldsymbol{X}_{m}(t)\right]\right\}\right\rangle .
$$


Ce facteur de structure présente la propriété remarquable que

$$
\lim _{q \rightarrow \infty} S(\boldsymbol{q})=1
$$

car l'existence même du désordre thermique impose que deux objets distincts finissent par apparaitre comme statistiquement indépendants lorsqu'on les considère à une échelle suffisamment locale et ce, même dans une structure cristalline. Il en résulte que les considérations développées à la Section 4 pour les sphères, les bâtonnets et les cylindres, valides stricto sensu seulement en solution diluée, sont donc en fait applicables à suffisamment grand vecteur d'onde, notamment avec des échantillons non orientés de phases cristal-liquides ou cristallines concentrées, pour autant que l'ordre orientationnel local soit très marqué dans le cas des cristaux-liquides.

\subsection{Structures ordonnées}

L'ordre de position est unidimensionnel dans les structures smectiques, bidimensionnel pour les structures colonnaires et tridimensionnel pour les structures cristallines. La description complète des allures très variées sous lesquelles se manifeste le facteur de structure dans ces différents cas est, pour une grande part, du ressort de la cristallographie (cf., parmi beaucoup d'autres, l'une ou l'autre des références [15]) et ne sera pas tentée ici. Une propriété générale de l'éq. (5.3) est, en l'absence de tout désordre dans la répartition des colloïdes dans l'espace, la présence de raies de Bragg, c'est-à-dire de valeurs de $\boldsymbol{q}$ pour lesquelles le facteur de structure prend une valeur très grande (à la limite, divergente pour $N \rightarrow \infty$ ), sa valeur étant au contraire très petite (et, à la limite, nulle) en dehors de ces positions localisées caractéristiques. Lorsque du désordre est modérément introduit dans la structure (fluctuations thermiques ou défauts), les raies de Bragg subsistent mais sont moins intenses et le facteur de structure acquiert des valeurs finies entre les positions de Bragg, pour finalement s'approcher asymptotiquement de la valeur 1 pour $q \rightarrow \infty$.

Ce comportement est ici illustré dans le cas, le plus simple qui soit, de la structure unidimensionnelle d'une phase lamellaire lyotrope ${ }^{4}$. La structure considérée est donc celle de $N$ bicouches planes de section $\mathcal{A}$ empilées sur une hauteur $L$ le long d'une direction $z$. Les positions des bicouches sont données par $\boldsymbol{X}_{l}(t)=\left[l d-u_{l}(t)\right] \hat{z}$ où $d$ est la période de l'empilement et $u_{l}(t)$ décrit l'écart de la $l^{\mathrm{e}}$ bicouche par rapport à la position requise pour un empilement strictement périodique. Le modèle n'est bien sûr pas très réaliste en ce qu'il suppose les bicouches planes, les fluctuations de courbure ne pouvant être considérées en raison de l'approximation initiale d'objets de forme fixe. Le facteur de structure devient dans ce cadre

$$
\mathrm{S}(\boldsymbol{q})=\frac{1}{N} \sum_{l, m} e^{-i q_{z} d(l-m)}\left\langle\exp \left[i q_{z}\left(u_{l}-u_{m}\right)\right]\right\rangle .
$$

Pour un empilement strictement périodique, les $u_{l}(t)$ sont tous nuls et le facteur de structure se réduit à la fonction d'interférence du réseau de $N$ traits

$$
\mathrm{S}(\boldsymbol{q})=\frac{\sin ^{2} N q_{z} d / 2}{N \sin ^{2} q_{z} d / 2}
$$

c'est-à-dire présente aux positions de Bragg $\boldsymbol{q}=\left(q_{B}^{(l)}, \boldsymbol{q}_{\perp}\right)$, régulièrement espacées dans la direction $z$ avec $q_{B}^{(l)}=2 l \pi / d$, des raies intenses et étroites, de hauteur $N$ et de largeur de l'ordre de $2 \pi /(N d)$ mais une valeur faible de l'ordre de $1 / N$ en dehors de ces positions. La prise en compte d'écarts à l'empilement périodique dus à la distorsion élastique de la phase lamellaire du fait des

\footnotetext{
${ }^{4}$ Le cas d'une phase hexagonale 2D est explicitement traité par une approche très voisine, cf. Réf. [16]. Par ailleurs, le site [17] fournit un programme permettant l'analyse de plusieurs situations, couramment rencontrées, de structures colloïdales ordonnées uni-, bi- ou tridimensionnelles.
} 


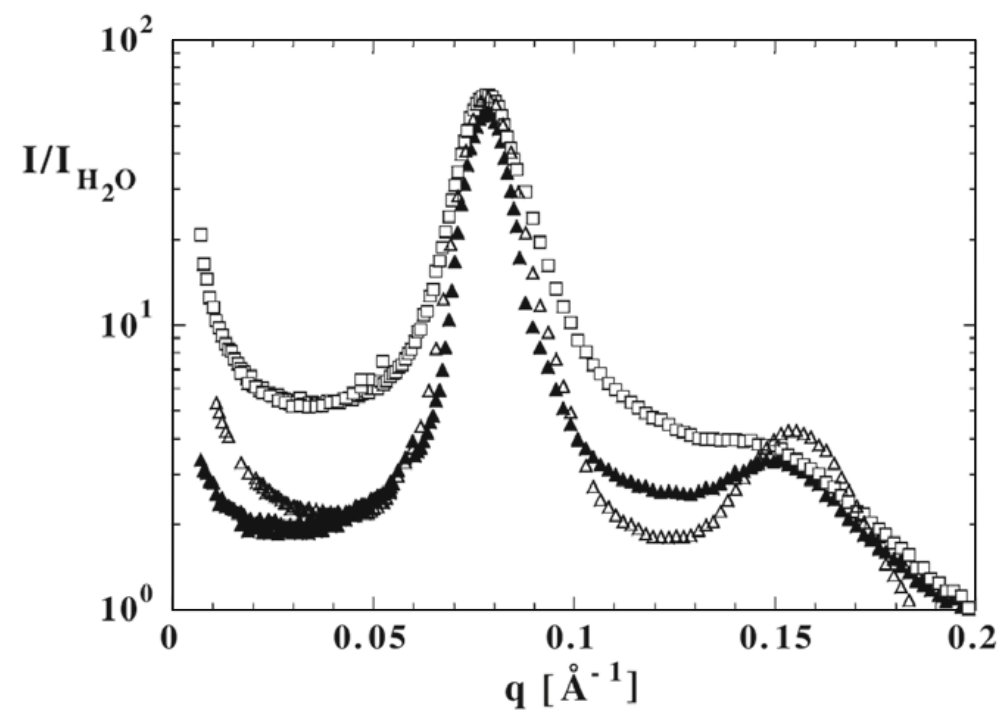

Figure 9. Spectre de diffusion des neutrons aux petits angles d'une phase lamellaire lyotrope «dopée» par un polymère hydrosoluble. La diminution de la constante élastique $\bar{B}$ qui accompagne l'augmentation de la teneur en polymères se traduit par une augmentation du signal diffusé à $q \rightarrow 0$, conformément à la description thermodynamique donnée Section 3.2.3, mais aussi par une augmentation de l'étalement des raies de Bragg, ainsi que du signal diffus entre raies de Bragg.

fluctuations thermiques fait intervenir, selon l'éq. (5.5), les fonctions de corrélation du type $\left\langle e^{\left[i q_{z}\left(u_{l}-u_{m}\right)\right]}\right\rangle$. L'expression de ces fonctions de corrélation dépend des constantes élastiques de la phase lamellaire, avec (pour $|l-m| \gg 1$ ) [18]

$$
\left\langle\exp \left[i q_{z}\left(u_{l}-u_{m}\right)\right]\right\rangle=\left[\pi e^{\gamma}|l-m|\right]^{-\eta q_{z}^{2} / q_{B}^{2}}
$$

où $\gamma \approx 0.57721$ est la constante d'Euler, $q_{B} \equiv 2 \pi / d$, la première position de Bragg et l'exposant $\eta$, qui contrôle en définitive la décroissance des corrélations de distorsions élastiques de bicouches distinctes, est donné par $\eta=q_{B}^{2} k_{B} T /(8 \pi \sqrt{K \bar{B}})$.

La décroissance en loi de puissance des corrélations de distorsions élastiques, éq. (5.7) est inhabituellement rapide pour une structure ordonnée et se trouve être la conséquence directe de la nature unidimensionnelle de l'ordre dans un cristal-liquide smectique. Les conséquences sur le facteur de structure, éq. (5.5), sont considérables puisque les raies de Bragg sont «étalées», avec un signal variant asymptotiquement au voisinage de la position de Bragg $q_{B}^{(l)}$ comme

$$
\mathrm{S}\left(q_{z}, \boldsymbol{q}_{\perp}=\mathbf{0}\right) \sim\left|q_{z}-q_{B}^{(l)}\right|^{-2+l^{2} \eta} .
$$

En outre, le facteur de structure rejoint beaucoup plus rapidement sa valeur limite de 1 aux grands vecteurs d'onde que dans le cas d'une structure dont l'ordre se déploie en dimension 2 ou 3. Ces deux propriétés sont mises à profit en diffusion aux petits angles pour déterminer, par l'analyse du facteur de forme de la bicouche, les paramètres structuraux de cette dernière [19, 20]. En se focalisant plutôt sur le facteur de structure, les interactions entre bicouches et leur flexibilité peuvent être étudiées : ces propriétés influent en effet sur la valeur des constantes élastiques $\bar{B}$ et $K$ et, par conséquent, sur la valeur de l'exposant $\eta$. Cette dernière application est illustrée de façon assez frappante par la Fig. 9 qui montre un étalement de la raie de Bragg d'ordre 1, ainsi qu'une moindre visibilité de la raie d'ordre 2 dans le cas d'une phase lamellaire lyotrope «dopée» par un polymère en concentration croissante dans 
le solvant [21]. De même, l'effet spécifique d'ions sur l'élasticité de phases lamellaires de membranes fortement chargées est étudié par cette méthode dans la Réf. [22].

\subsection{Structures désordonnées}

En l'absence d'ordre de position, le facteur de structure ne s'éloigne jamais beaucoup de sa valeur asymptotique de 1, sauf peut-être dans la limite $q \rightarrow 0$ lorsque les interactions, attractives ou répulsives, influent significativement sur la compressibilité osmotique. Du fait de l'absence des raies de Bragg, ses variations avec le vecteur d'onde sont également beaucoup moins abruptes que dans le cas des systèmes ordonnées. Par ailleurs, si l'on peut supposer que tous les constituants sont équivalents en moyenne (ce qui impose qu'une éventuelle polydispersité soit négligeable), l'éq. (5.3) est usuellement reformulée comme

$$
\mathrm{S}(\boldsymbol{q})=\frac{1}{N}\left\{N+N(N-1)\left\langle\exp \left[i \boldsymbol{q} \cdot\left(\boldsymbol{X}_{2}-\boldsymbol{X}_{1}\right)\right]\right\rangle\right\}
$$

puisqu'il y a $N(N-1)$ couples d'objets distincts pour $N$ objets présents dans le volume irradié. Il est courant dans ce contexte d'introduire la fonction de corrélation de paires $g(\boldsymbol{X})$ pour donner le facteur de structure sous la forme [23]

$$
\mathrm{S}(\boldsymbol{q})=1+\frac{\phi}{\mathcal{N} v} \int d^{3} \boldsymbol{X} e^{i \boldsymbol{q} \cdot \boldsymbol{X}}[g(\boldsymbol{X})-1] .
$$

Les méthodes habituelles de la théorie des liquides simples, appliquées au contexte des systèmes colloïdaux, fournissent une description souvent satisfaisante de la fonction de corrélation de paires et, par voie de conséquence, du facteur de structure en termes d'interactions, attractives aussi bien que répulsives, entre éléments dispersés [24]. Ce point est traité en amples détails, par Luc BeLloni, plus loin dans ce volume.

\section{Remerciements}

Il m'est agréable de remercier les organisateurs de l'école thématique «Neutrons et matière molle», ainsi que ceux des $17^{\text {es }}$ Journées de la diffusion neutronique - et plus spécialement Fabrice Cousin et Julian OberdissE. Sans leurs sollicitations, souriantes autant que courtoises, ni la version orale, ni la présente version écrite de ce cours n'auraient vu le jour.

\section{Références}

[1] On pourra consulter le site http://www.ncnr.nist.gov/resources/n-lengths/ pour de plus amples détails

[2] Voir http://www-llb.cea.fr/fr-en/pdf/TPA.pdf

[3] G. Porod, in Small Angle X-ray Scattering, p. 17, éd. O. Glatter et O. Kratky, Academic Press, Londres, 1982

[4] L.S. Ornstein et F. Zernike Proc. Acad. Sci. Amsterdam 17 (1914) 795

[5] L.D. Landau et E.M. Lifshitz, in Physique statistique, $4^{\mathrm{e}}$ édition, Mir, Moscou, 1994

[6] P. Flory, in Principles of Polymer Chemistry, Cornell University Press, Ithaca, 1953

[7] J. des Cloizeaux J. Phys. France 36 (1975) 281

[8] M. E. Cates, D. Roux, D. Andelman, S. T. Milner et S. A. Safran Europhys. Lett. 5(1988) 733

[9] F. Nallet, D. Roux et S.T. Milner J. Phys. France 51 (1990) 2333

[10] F. Castro-Roman, L. Porcar, G. Porte et C. Ligoure Eur. Phys. J. E 18 (2005) 259

[11] Le site http://kur.web.psi.ch/sans1/SANSSoft/sasfit.html permet d'accéder à une collection très complète de modèles géométriques, ainsi qu'aux outils numériques associés pour le paramétrage de données expérimentales 
[12] Beaucoup des propriétés utiles des fonctions de Bessel sont données sur le site http: //mathworld.wolfram.com/BesselFunctionof theFirstKind.html

[13] P. Debye, J. Chem. Phys. 14 (1946) 636

[14] J. Teixeira, J. Appl. Cryst. 21 (1988) 781

[15] Quelques références pour ce très vaste sujet: G. Friedel, in Leçons de cristallographie professées à l'université de Strasbourg, Berger-Levrault, Paris, 1926. A. Guinier, in Théorie et pratique de la radiocristallographie, Dunod, Paris, 1956. B.K. Vainshtein, in Modern Cristallography I, Springer-Verlag, Berlin, 1981.

[16] N. Freiberger et O. Glatter J. Phys. Chem. B 110 (2006) 14719

[17] Le site http://www.chemie.uni-hamburg.de/pc/sfoerster/software.html permet de télécharger un programme pour l'analyse de spectres obtenus avec des systèmes colloïdaux structurés selon une, deux ou les trois directions de l'espace

[18] A. Caillé, C. R. Hebdo. Acad. Sci. Paris B274 (1972) 891

[19] G. Pabst, M. Rappolt, H. Amenitsch et P. Laggner Phys. Rev. E 62 (2000) 4000

[20] N. Kučerka, Y. Liu, N. Chu, H.I. Petrache, S. Tristram-Nagle et J.F. Nagle Biophys. J. 88 (2005) 2626

[21] M.-F. Ficheux, A.-M. Bellocq et F. Nallet Eur. Phys. J. E 4 (2001) 315

[22] G. Brotons, M. Dubois, L. Belloni, I. Grillo, T. Narayanan et Th. Zemb J. Chem. Phys. 123 (2005) 024704

[23] J.-P. Hansen et I.R. Mac Donald, in Theory of simples liquids, Academic Press, Londres, 1976

[24] L. Belloni J. Phys. Condens. Matter 12 (2000) R549 